\title{
40. RADIO ASTRONOMY (RADIO ASTRONOMIE)
}

PRESIDENT: Y.N. Parijskij.

VICE-PRESIDENT: $H$. van der Laan.

ORGANIZING COMMITTEE: E.J. Blum, D.S. Heeschen, A.D. Kuzmin, D. McLean, T.K. Menon, P. Mezger, H. Palmer, B. Robinson, B.M. Zuckerman.

\section{GENERAL REMARKS}

Thousands of papers in the field of radio astronomy have appeared during the last three years. Even simple enumeration of the major achievements will take much more space than it has been allocated to Commission 40 . Therefore, taking into account the tendency of the previous years, much greater attention in the present report has been paid to the actual material and references (mainly of observational and methodological character). As to the interpretation of observations, much overlapping has occurred with Commissions 10 and 34. Solar radio astronomy had to be excluded from the report of Commission 40 in view of its practically complete overlapping with the report of Commission 10. Physical interpretation of insterstellar medium observations carried out by radio techniques during the three years has been included in the report of Commission 34. Even in this case we have been forced, as in the previous years, to introduce some additional abbreviations of the most widely used journals in the sections containing a great number of references. These abbreviations are given below.

\section{ABBREVIATIONS}

$A A=$ Astronomy and Astrophysics

Act Astr $=$ Acta Astronomica

$A J=$ Astronomical Journal

$A J P=$ Australian Journal of Physics

$A N=$ Astronomische Nachrichten

$A O=$ Astronomical Observatory

$A p J=$ Astrophysical Journal

$A L=$ Astrophysical Letters

$A S S=$ Astrophysics and Space Science

Astrofiz. = Astrofizika

$A R A A=$ Annual Reviews of Astronomy and Astrophysics

$A Z h=$ Astronomicheskij Zhurnal

$B A A S=$ Bulletin of the American Astronomical Society

$C I A L=$ Coll. Int. Astrophys. Liège

$C R=$ Comptes Rendus de I'Academie des Sciences, Paris

$I E A M=$ First European Regional Meeting in Astronomy (Athens)

$H A=$ Highlights of Astronomy

$I A U$ Circ. = IAU Circular

$I A U$ Symp. = IAU Symposium

$I E E E=$ Transactions of the Institute of Electrical and Electronic Engineers

$I z v S A O=$ Izvestiya Spetsialnoj Astrofizicheskoj Observatorii

$I V U Z R=$ Izvestiya Vysshikh Uchebnykh Zavedenij

$J R A S C=$ Journal of the Royal Astronomical Society of Canada

$M N=$ Monthly Notices of the Royal Astronomical Society

$M R A S=$ Memoires of the Royal Astronomical Society

Nat $=$ Nature

NatPS = Nature Physical Science

$O b s=$ Observatory 
$P A S A=$ Proceedings of the Royal Astronomical Society of Australia

$P A S J=$ Publications of the Astronomical Society of Japan

$P A S P=$ Publications of the Astronomical Society of the Pacific

$P R L=$ Physical Review Letters

Soviet Astron. = Soviet Astronomy

Sci $=$ Science

$S R=$ Space Research

Sup $=$ Supplement

$V \boldsymbol{A}=$ Vistas in Astronomy

\section{CONTINUUM RADIATION FROM THE GALAXY}

\section{A. General Background}

New high sensitivity and high resolution maps have been published at a number of frequencies $(40,50,21,1,28,7,18,19,75,72,71,70,66,64,51,36)$, and spectral indices have been improved. The most. important results have appeared after nonthermal radio and X-Ray surveys were compared. Now we have a strong and very direct confirmation of the old hypothesis on the secondary production of ultra-relativistic electrons responsible for long wavelength radio emission of our Galaxy. Very low $(61,47,4,3,12)$ and very high $(5,39)$ frequency observations of the Milky Way should be mentioned specially, both being sensitive to the amount of ionized gas. Results of surveys of continuum emission polarization can be found in $(77,9,48)$, the structure of magnetic field being analysed in $(78,73,55)$.

\section{B. Supernovae}

A large number of very detailed maps have been produced $(37,34,33,67,24,69,15,42$, $38,15,17)$, many of these containing important polarization information $(52,4,60,59,53)$. Statistics and evolution are discussed in $(22,65,43,35,20,2,14,13)$. A good review of the present state of the whole problem can be found in (61).

\section{Galactic Centre}

Much better high resolution maps have appeared due to cooperation between observatories $(68,6,30,31,44,49,54,56,57,63,25,26,76,11,10)$. An attempt to find pulses from Sgr $A$ sources has been made, but without success $(27,23,41,42)$. New important steps have been made by discovering SNR (Sgr A, West) and broad recombination line in Sgr A. The review of all new observations of this complex region can be found in (57).

\section{REFERENCES}

1. Abramenko et al. 74Soobshcheniya SAO12, 43.

2. Akhundova et al. 74Astrofiz10,85.

3. Alexander et al. $74 A J 79,777$.

4. Antonow. 73IVUZR16, 759.

5. Ariskin et al. 74Astron. Tsirk. No 833, 6 .

6. Ariskin et al. $74 A J 51,5,916$.

7. Ariskin. $73 A J 50,2,283$.

8. Baker. $74 A p J 194,271$.

9. Baker et al. $74 M N 167,581$.

10. Balick et al. $74 A p J 192,325$.

11. Berulis et al. $73 A J 50,2,270$

12. Balick et al. $74 A$ pJ194, 265.

13. Brown. $73 A$ pJ180, 359.

14. Cavallo et al. $74 A S S 30,409$.

15. Chevalier et al. $74 A p J 192,454$.
16. Clark et al. 73Nat 246, 5427.

17. Clark et al. 75AJP Sup37, 75.

18. Clark et al. 75AJP Sup37, 1.

19. Cowsik et al. 74 Mitt.Astron.Ges. 35 , 224.

20. Cowsik et al. 73 13th Int.Cosm.Ray. Conf.Denver. Colo. 1, 414.

21. Dellaporta. 74LAU Symp, 189.

22. Day. 73CSIRO Div.Radiophys. Res. Activ.

23. Dickel et al. $74 B A A S 6,314$.

24. Dube et al. 73NatPS245, 141, 17.

25. Duin et al. $74 I A U$ Symp.60, 355 .

26. Dulk et al. $72 A J P 25,429$.

27. Dulk et al. 74Nat 248, 5443, 33.

28. Edwards et al. 74Nat 247, 5441, 444. 
29. Felli et al. $73 A A 29,1,129$.

30. Fujimoto et al. 74PASJ26, 399.

31. Gezari et al. 73ApJ179, L67.

32. Gordon 74IAU Symp.60, 477.

33. Green $74 A A$ Sup18, 2, 267.

34. Gull $73 M N 162,135$.

35. Gull $75 M N 171,237$.

36. Gulliford 74ASS31, 241.

37. Haslam et al. 74AA Sup13, 359.

38. Hill $74 M N 169,59$.

39. Hirabayashi $74 I A U$ Symp.60, 353.

40. Hirabayashi 74IAU Symp.60, 187.

41. Hirabayashi $74 P A S J 26,2,263$.

42. Hughes et al. 73Nat 242, 5393, 105.

43. Hughes et al. 74IAU Symp.60,401.

44. Johnson 74ApJ194, 337.

45. Jones $74 A A 30,1,37$.

46. Jones et al. 74AJP5, 687.

47. Sofue 73PASJ25, 207.

48. Zhouckl 73IVUZR16, 5, 754.

49. Kapustin et al. 73IVUZR16, 1325.

50. Kapitzky et al. 74ApJ188, 27.

51. Keen $73 A A 24,2,229$.

52. Kelly $74 M N 33$.

53. Kundu et al. 74AJ79, 132.

54. Kundu et al. $73 A J 78,170$.

55. Little 74IAU Symp.60, 491.

56. Manchester $74 A p J 188,644$.
57. Mezger 74ESO/SRC/CERN Conf.Res. Program, New Large Telescopes Geneva Proc.

58. Mezger et al.

59. Milne et al. $74 A J P 27,549$.

60. Milne et al. 74IAU Symp.60, 335.

61. Mills 74IAU Symp.60, 311 .

62. Milogradov-Turin et al. $74 M N 161$, 269.

63. Mongain 73 NatPS242, 122, 136 .

64. Rougoor et al. 74AA33, 3, 459.

65. Shakesháft 74IAU Symp.60, 185.

66. Shklovskii 74IAU Symp.60, 373.

67. Sironi $74 M N 166,2,345$.

68. Slee et al. 74IAU Symp.60, 347.

69. Soboleva 74AZh51, 1341.

70. Strom et al.

71. Price 74IAU Symp.60, 637 .

72. Price $74 A A 33,33$.

73. Taylor 73Proc. IEEE61, 4, 469 .

74. Vajsberg $74 A Z h 51,922$.

75. Velusamy et al. $74 A A 32,375$.

76. Webster $74 M N 166,2,355$.

77. Whiteoak et al. $73 A L 13,14,205$.

78. Wilkinson $73 M N 163,2,147$.

79. Wilkinson et al. $74 M N 167,593$.

\section{H II Regions Radio Emission}

\section{Gaseous Nebulae}

During the last three years (73-75) observations of continuum radio emission from H II regions in a vast range of wavelengths $(1 \mathrm{~mm}$ to $75 \mathrm{~cm}$ ) have been carried out. In comparison with the preceding three years a significant increase in the amount of observational data obtained at $\mathrm{mm}$ wavelengths is quite evident. Not only bright giant $\mathrm{H}$ II regions but also faint optically visible nebulae have been investigated. The study of brightness distribution of different types of H II regions with high and intermediate resolution goes on, however, some new features may be distinguished. Special attention has been paid to the statistical study of relations between compact and large-scale structures within $H$ II regions, also between compact $\mathrm{H}$ II regions, $\mathrm{OH} / \mathrm{H}_{2} \mathrm{O}$ masers and bright infrared emission sources. In this connection it should be emphasized that investigation of molecular lines and infrared emission of $\mathrm{H}$ II regions has been very intensive.

The intensive study of recombination radio lines of excited hydrogen, helium and carbon, and maybe some heavier elements is continued. Statistical investigation of electron temperatures obtained from the data on recombination radio lines has revealed that the electron temperature of $\mathrm{H}$ II regions depends on the galactic radius.

Some studies of the effect of electron temperature and density fluctuations, and gradients on the continuum and recombination line emission of $H$ II regions have been performed.

The first high resolution survey of continuum radiation from extragalactic $\mathrm{H}$ II regions in the nearby galaxy $M 33$ has been made.

\section{Planetary Nebulae}

The intensive study of radio continuum in $\mathrm{cm}$ and $\mathrm{dm}$ wavelength ranges is continued. Detailed radio maps of a great number of planetary nebulae have been obtained in the continuum with a resolution as small as $2^{\prime \prime}$. In a number of planetary nebulae recombination radio lines of excited hydrogen have been observed. 


\section{Radio Stars}

Radio emission of several new objects of MWC 349 type, peculiar stars with forbidden emission lines and large infrared excesses, have been revealed. It seems that new-type objects have appeared - Radio ETELS (Early-Type Emission Line Stars). Radio emission is supposed to be generated in vast gaseous envelopes, some kind of protoplanetary nebulae formed as a result of mass loss by the central star.

Complex investigation of X-ray, optical and radio emission of X-ray stars is continued.

\section{H II REGIONS}

1. Surveys. Flux densities

\begin{tabular}{|c|c|c|}
\hline 1.1 & Amirkhanyan et al. & \\
\hline 1.2 & $\begin{array}{l}\text { 73Astron. Circ. } 802,7 . \\
\text { Churchwell et al. }\end{array}$ & 14.4 \\
\hline & $73 A A 23,117$ & 2.695 \\
\hline 1.3 & $\begin{array}{l}\text { Terzian et al. } \\
73 P A S P 85,806 .\end{array}$ & 2.175 \\
\hline 1.4 & $\begin{array}{l}\text { Blair et al. } \\
74 P A S P 86,599(\mathrm{a}) .\end{array}$ & \\
\hline 1.5 & Clark et al. & \\
\hline 1.6 & $\begin{array}{l}74 A J P 27,713 . \\
\text { Clegg et al. }\end{array}$ & 0.408 \\
\hline 1.7 & $\begin{array}{l}74 \text { Nat } 249,530 . \\
\text { Bfanov et al. }\end{array}$ & 77.5 \\
\hline 8 & $74 A Z h 18,4$ & 77.5 \\
\hline 1.9 & $\begin{array}{l}74 A A \text { Sup16, } 43 . \\
\text { Felli et al. }\end{array}$ & 0.408 \\
\hline & 74ASS26, 901. & 1.400 \\
\hline 1.10 & Klein $74 A J 79,139$. & 21.84 \\
\hline 1.11 & Rather $74 P A S P 86,601$ & 21.4 \\
\hline 1.12 & $\begin{array}{l}\text { Zabolotnyj et al. } \\
\text { 75Pisma AZh1, } 1.14\end{array}$ & $(30$ \\
\hline
\end{tabular}

2. Surveys. Structure

2.1 Berulis et al. 73 Soviet Astron.17. $\quad 36.5$

2.2 Israel et al. $73 A A 27,143$. 1.4

2.3 Felli et al. $74 A A 31,431$. $\quad 8.085$

2.4 Turner et al. 74ApJ194, 279.

2.5 Cogdell 75ApJ196, 363.

2.6 Wink et al.75AA38, 109.

3. Brightness distributions of single H II regions. Overall structure.

3.0 Bogod et al. 73 Soviet Astron. 17, $47=$ AZh52, 72 .

3.1 Ellis. 73PASA2, 158.
150

2.695

8.085

3.2 Johnston et al. $73 A J 78,235$.

3.3 Jones 73AJP26, 545.

3.4 Kaifu et al. 73PASJ25, 129.

3.5 Kislyakov et al.

73 IVUZR16, $774 . \quad$ OriA

3.6 Rarijskij et al.

73IVUZR16, 778 . M1-19

3.7 Terzian et al. 73 AJ78, 804.

3.8 Velusamy et al. $73 A J 78,31$.

Omega

Car Neb

OriA

Cyg X

S101

3.9 Ade et al.74ApJ189, 123.

M42,DR-21

3.10 Baars et al. 74IAU Symp.60, 219.

3.11 Caswell et al. 74AA32, OriA, OriB 209.

IC434

3.12 Elias et al. 74PASP86, 599.

3.13 Scalize et al. 74Nat225, 663.

DR-21

Car Neb

3.14 Chaisson et al. 75ApJ199, 647.

Trifid Neb

3.15 Gardner et al. 75MN171, 29P. G0.55-0.85

3.16 Werner et al. $75 A p J 199$, L185. DR-21

3.17 Goudis 75ASS33, 103. IC1 318

3.18 Zeilik III et al. G45.1+0.1 $75 A p J 199,401$.

$\mathrm{G} 45.5+0.1$

3.19 Walmsley et al. $75 A A 41,121$.

S206, 209

\section{Compact structure in $H$ II regions}

4.1 Harris $73 M N 162,5 \mathrm{P}$. DR-21

4.2 Martin 73MN163, 141. NGC7538

4.3 Martin 73Obs93, 164.

4.4 Sullivan III et al. 73AA29, 369.

4.5 Broderick et al. $74 A p J 192,343$.

4.6 Harris $75 M N 170,139$. K3-50

W3

G351.6-1.3 M17, OriA Gum Neb 
5. Compact $\mathrm{H}$ II regions associated with $\mathrm{OH} / \mathrm{H}_{2} \mathrm{O}$ masers and $I R$ sources

5.1 Baldwin et al.73Nat 241, 38

5.2 Goss et al. 73AA29, 435.

5.3 Matsakis $73 B A A S 5$, 450.

5.4 Winnberg et al. 73NatPS243,78.

5.5 Brown 74APJ194,L9.

5.6 Harris 74MN166, 29.

5.7 Wendker et al. 74AA33, 157.

6. Compilative and review papers, discussions

6.1 Angerhofer et al. 73Mitt. Astron.Ges. 32, 269.

Cat.opt.vis. $\mathrm{H}$ II reg.

6.2 Wilson et al. 75Lecture Notes Phys.42, Springer.

6.3 Balick et al. 74PASP86, 616.

$\mathrm{W} 3(\mathrm{OH})$

ON-2

OHO739-1.4

M78,S269

$1 \mathrm{C} 2162$

$\mathrm{ON}-1$

OH069.5-10

AFCRL8092992

6.4 Churchwell. 74IAU Symp. 60,195

ture

6.5 Habing 74Proc. NATO Adv. review Study Inst.91.

6.6 Terzian $74 V A 16,279$. review

6.7 Goudis 75ASS35, 409 . OriA

6.8 Goudis 75ASS36, 79 . OriA

6.9 Goudis $75 A S S 36,105 . \quad$ OriA

6.10 Gorschkov et al. $75 A Z h 52,538$.

7. Electron temperature in $H I I$ regions

7.1 Pyatunina et al. 73AZh50, 507.

7.2 Simpson 73PASP85, 479.

7.4 Churchwell et al. 75AA38, 451.

7.5 Pyatunina $75 I z v . S A O 7$, 101.

Tedistr

OriA

Te-gal.rad. dep.

$\mathrm{T}_{\mathrm{e}}$ distr.

8. Spectra of $H I I$ regions

8.1 Salem et al. $74 M N 167,493$.

8.2 Salem 74MN167, 511 .

8.3 Olnon 75AA39, 217. (see also 7.1, 6.9).

9. Infrared emission of $H$ II regions

9.1 Frogel et al. 72ApJ178, S138,152, 667.

270 .

9.2 Wynn-Williams et al. $72 M N 160,1$.

9.3 Becklin et al. 73ApJ182, L7.

W3

OriA
9.4 Becklin et al.

$73 A L 13,147$.

9.5 Becklin et al.

$73 A p J 182$, L125

9.6 Emerson et al.

$73 A p J 184,401$.

9.7 Erickson et al.

73ApJ183, 535 . OriA

9.8 Johnston $73 A p J 180, \mathrm{~L} 7$.

9.9 Persson et al.

73BAAS5, 318.

9.10 Rieke et al.

$73 A p J 183$, L67.

9.11 Swift et al.

73IAU Symp.52, 453 . OriA

9.12 Wynn-Williams et al.

73IAU Symp.52, 459.

9.13 Aannestad. 74BAAS6, 263.

9.14 Beetz et al. 74AA34, 335.

9.15 Bergeat et al. 74AA30, 181.

9.16 Dyck et al. 74ApJ194, 57.

9.17 Fazio et al. $74 A p J 192$, L23.

9.18 Frogel et al. 74ApJ192, 351.

9.19 Grasdalen $74 A p J 193$, 373.

9.20 Harper $74 A p J 192$, 557.

9.21 Houck et al. 74ApJ193,L139.

9.22 Lemke et al. 74AA32, 231.

9.23 Lunel et al. 74AA34, 299.

9.24 Mezger et al. 74AA32, 269.

9.25 Olthof et al. 72IEAM2, 243.

9.26 Panagia 74ApJ192,221.

9.27 Pipher et al. 74ApJ193, 283

9.28 Pipher et al. 74BAAS6, 335

9.29 Schaak et al. 74BAAS6, 329

9.30 Ward et al. 74BAAS6, 329(a).

W3

DR-21

OriA

OriA

Copm.IRsource.

W12

M42,NGC

2024

OriA

OriA, map

S157

G30. 8-0.0

G29.9-0.0

OriA

OriA

9.31 Ward et al.

74Nat 252, 27.

9.32 Wynn-Williams et al. 74PASP86, 5.

9.33 Wynn-Williams et al. $74 A p J 187,473$.

9.34 Wynn-Williams 74IAU Symp.60, 259.

9.35 Soifer et al. $74 A p J 191$, L83.

9.36 Fazio et al.75ApJ199, L117.
OriA,

NGC2024

W3 
9.37 Harvey et al. 75ApJ196, L31.

9.38 Soifer et al. 75ApJ199, 663.

9.39 Ward. $75 A p J 200, \mathrm{~L} 41$.

OriA spectrum

9.40 Thum et al. 75AA41, 467. DK-21 (see also $3.9,5.3,5.5,6.7$ )
10. $H$ II regions in nearby galaxies

$\begin{array}{lll}10.1 & \text { Israel et al. } & \\ & 74 A A 32,363 . & 1.415 \\ 10.2 & \text { Spencer et al. 74IAU Symp. } \\ & 60,229 . & \\ 10.3 & \text { Andrew. 75AA39, 421. } & \mathrm{H}_{2} \mathrm{O} \mathrm{em} \\ 10.4 & \text { Israel } \text { et al. } & 0.61,1.415, \\ & 75 A A 40,421 . & 4.995, \mathrm{M} 101\end{array}$

PLANETARY NEBULAE

1. Surveys. Flux densities

1.1 Johnston. 73CIAL18, 121. 31.85

1.2 Sistla et al. 73BAAS5, 424(a).

15.5

1.3 Terzian et al. 73AJ78, 875

1.4 Cahn et al. 74AJ79, $128 . \quad 2.7,8.1$

1.5 Sistla et al. $74 A p J 192,165$.

15.5

1.6 Milne et al. 75AA38, $183 . \quad 5.0$

1.7 Scott $75 M N 170,487$. 5.0

\section{Structure}

2.1 Balick et al. 73ApJ182, L117.

2.2 Kaftan-Kassim 73CIAL18, 129.

2.3 George et al. 73BAAS5, 424.

2.4 Scott. $73 M N 161,35 \mathrm{P}$.

2.5 Terzian et al. $73 A p J 188$, 257.

2.6 Georfe et al. 74AA35, 219.

2.7 Sistla et al. $74 B A A S 6,425$.

2.8 Willis et al. $74 A A 36$, 455.

NGC7027

1C2149, NGC 6543,7662

NGC7027

NGC40, 6543 7662

NGC3568

NGC6543
2.9 Sistla et al. 74MN166, 17.

2.10 Purton et al. 75ApJ195, 479.

ICZ149

M2-9

3. Infrared emission

3.1 Becklin et al. 73AL15, 87. NGC7027

3.2 Danziger 73ApJ184, L29. NGC6302

3.3 Cohen et al. 74ApJ193, 401.

survey

3.4 Khromov 74AZh51, 335.

3.5 Telesco et al. $74 B A A S 6$, 464.

NGC7027

3.6 Boksenberg et al. $75 M N 171,395$.

NGC6543

3.7 Danziger 75AA38, 475. NGC6210

3.8 Khromov 75Pisma AZh8, 16.

3.9 Merrill et al. $75 A p J 200, \mathrm{~L} 37$.

evol.eff.in

NGC7027

4. Compilative and review papers

4.1 Higgs $73 M N 161,313 . \quad$ spectra

4.2 Higgs 73CIAL18,89. spectra

4.3 Higgs $73 C I A L 18,101$.

4.4 Miller 74ARAA12, 331, review

4.5 Thomson $74 V A 16,309$. review

\section{RADIO STARS}

A. Radio emission of $O B$ stars

1. Observations

1.1 Altenhoff et al. 73 Nat 241, 37.

1.2 Altenhoff et al. $731 A U$ Circ. 2549.

1.3 Altenhoff et al. 73IAU Circ. 2569.

1.4 Baldwin et al. 73Nat 241, 38.
1.5 Feldmann et al. $73 I A U$ Circ. 2543.

1.6 Feldman et al. $73 I A U$ Circ. 2549.

V1016Cyg HD167362, VY2-2

L Ori,HBV475, MWC349

1.7 Feldman et al. 73IAU Circ. $2560 . \quad$ M2-9

1.8 Feldman et al.

M1-11

V1016Cyg 73 NatPS245, 7.

HD37806

MWC137, LkHL101

1.9 Feldman et al. 73 NatPS245, 39.

MWC957.

1.10 Gregory et al. 73NatPS242, 101.

MWC349 

1.11 Gregory et al.
73IA U Circ. 2556. R Aquarii
1.12 Greenstein.
73ApJ184, L23
1.13 Hjellming et al.
73 NatPS242,84.
1.14 Hughes et al. 73NatPS242, $116 . \quad$ RY Scuti
1.15 Marsch et al.
$73 I A U$ Circ. 2565.
M1-11,
HD37806
1.16 Morrow et al.
73 IAU Circ. 2567.
1.17 Purton et al.
73 NatPS245, 5.
1.18 Purton et al. 73NatPS245, 6.
1.19 Seaquist $e t$ al. 73IAU Circ. 2563
1.20 Wendker et al. 73 NatPS245, 118.
1.21 Gregory et al.
i. 74Nat 247, 532.
1.22 Wright et al. 74 Nat 250, 715.
MWC957
V1016Cyg
VY2-2,
HD167362
V1016Cyg
VY2-2
P Cyg
R Aquarii
Hen 1044

\section{Interpretation}

2.1 Barrata et al.74ApJ187, 651 .

2.2 Braes 74IAU Symp. 60, 377.

2.3 Ciatti et al. 74AA34, 181 .

2.4 Fitzgerald et al. 74ApJSup28, 147.

2.5 Marsch et al. 74JRASC68, 265(a).

2.6 Purton et al. 74BAAS6, 457.

2.7 Ciatti et al. 75AA38, 435 .

2.8 Wright et al. $75 M N 170,41$.

B. Radio emission of $X$-ray binaries

1. Observations

1.1 Hjellming et al.72ApJ178, L139.

Per

1.2 Aller et al. 73NatPS245, 40.

Cyg X-3

1.3 Bash et al. 73NatPS241, 93.

Cyg X-3

1.4 Braes et al. 73NatPS242, 66.

Cyg X-3

1.5 Gregory. 73JRASC67, 199. Cyg X-3

1.6 Gregory et al. 73BAAS5, 394(a). Cyg X-3

1.7 Hjellming et al. 73Nat 242, $250 . \quad$ Per

1.8 Hjellming et al. 73 NatPS243, 81.

1.9 Icke. 73NatPS244, 132.

1.10 Pooley et al. 73Nat 244, 270.

1.11 Becklin et al. 74ApJ192, L119. Cyg X-3

1:12 Daishido et al. 74 Nat 251, 36.

Cyg X-2

Cyg $X-3$

Per

Cyg X-3
1.13 Gibson et al.74PASP86, 652 .

CC Cas,

AR Lac

1.14 Hjellming et al.

$74 A p J 194, \mathrm{~L} 13$.

Cyg X-3

1.15 Hughes et al.74ApJ191, 749.

Cyg X-3

1.16 Hughes et al. 74IAU Symp. 60, 407. Cyg X-3

1.17 Kawano et al.74Journ.Rad. Res.Lab.Japan 21, $85 . \quad$ Sco X-1

1.18 Marsch et al. 74Nat248, 319.

Cyg X-3

1.19 Marsch et al. 74ApJ192, 697.

Cyg X-3

1.20 Seaquist et al. 74Nat251, 394.

Cyg X-3

1.21 Braudt et al. 75ApJ197, 443.

Sco X-1

1.22 Canizares et al. 75ApJ197, 457.

1.23 Clark et al. 75ApJ198, L123.

Sco X-1

1.24 Gregory et al. $75 \mathrm{ApJ199,}$ 467.

1.25 Kawano et al. 75PASJ27, 191. Per

Cyg X-3 Cyg X-3

1.26 McEllin. 75MN170, $1 \mathrm{P}$.

2 Models, review papers

2.1 Bahcall et al. 73NatPS244, 135.

2.2 Braes et al. 73IAU Symp.55, 86.

2.3 Hjellming 73IAU Symp.55, 48.

2.4 Hjellming 73Sci182, 1089.

2.5 Hughes et al. 73JRASC67,199(a).

2.6 Peterson 73Nat 242, 173.

2.7 Gregory et al. 74ApJ194, 715 .

2.8 Marsch et al. 74BAAS6, 280.

2.9 Pringle 74Nat 247, 21.

2.10 Ramaty et al. 74ApJ187,61.

C. Radio emission of flare stars

1. Hughes et al. 73NatPS246, 111.

2. Hughes et al. 73IAU Circ. 2582.

3. Spangler et al. $74 A p J 190$, L129.

R Aquilae

R. Aquilae

YZ YMa,

AD Leo

V3710ri,

Wolf 424

4. Spangler et al. 74ApJ194, L43.

AD Leo

5. Tovmassian et al. 74 Astrofiz10, 337.

D. Stars with extended coronae

1. Wendker et al. 73NatPS245, 118.
2. Landini et al. $73 S R, X I I I 2$, 859. 


\section{E. PULSARS}

At the present time the initial stage of 'pulsar accumulation' is being replaced step by step by the stage of their thorough investigation. The state of the matter is reflected in the papers published during the period under review (1),(2). The principal observational and some theoretical results are presented in (3). The most complete catalogue, including 147 pulsars can be found in (4). The bibliography presented below contains references mainly to observational works.

Information on new pulsar discoveries is contained in (5)-(11). The most remarkable fact is the discovery of a pulsar in a binary system (12).

Pulsar distances, proper motions, positions are found in (13)-(15). Pulsar periods, period derivatives are in (16)-(22). Period discontinuities in slow pulsars: (23), (24). Pulse profiles, their changes and variability: (25)-(33). Spectra, polarization in pulses, subpulses, precursors: (34)-(65). Pulsars and study of interstellar medium, halos, scintillations etc: (67)-(81). VLBI results are in (82)-(84).

As to the nature of pulsar phenomenon, it is only generally accepted now that a pulsar is a neutron star and mechanisms of radio emission are coherent. The lack of complete clarity is mainly due to the insufficiency of knowledge of matter in the extreme conditions. The general characteristic of the magnetosphere of pulsars is presented in (85). The radiation mechanisms suggested and their discussion can be found in (86)-(92). A survey on the inner structure of neutron stars is in (93).

\section{REFERENCES}

1. Manchester 73Proc. IEEE61, 1205.

2. Sieber $73 A A 28,237$.

3. ter Haar 75Contemp.Phys 16, 243.

4. Taylor et al. $75 A J 80,794$.

5. Davies et al. 73 NatPS244, 84.

6. Komesaroff et al. 73IAU Circ. 2505, 2563.

7. Bosignori-Facondi $73 A A 27,67$.

8. Crovisier $73 A L 13,225$.

9. Hulse et al. 74ApJ191, L59.

10. Komesaroff et al. $74 A L 15,169$.

11. Mohanty et al. $75 M N 171,17 \mathrm{P}$.

12. Taylor et al. 74IAU Circ. 2704,2712 , 2714, 2720, 2731.

13. Gonzàlez et al. $73 A L 13,229$.

14. Vaughan et al. 74MN168, 361.

15. Manchester 74ApJ189, L119.

16. McCulloch et al. $73 A L 14,169$.

17. Michel 73BAAS5, 425 .

18. Graham et al. 73 NatPS242, 86.

19. Sieler et al. $73 A L 13,225$.

20. Richards et al. 74Nat 251, 37.

21. Zyne $75 M N 171,579$.

22. Mert 73BAAS5,321.

23. Shabanova $74 A Z h 51,931$; Soviet Astron. 18, 5.

24. Manchester et al. 74ApJ191, L63.

25. Smith $73 M N 161,9 \mathrm{P}$.

26. Hankins $73 A p J 181, \mathrm{~L} 49$.

27. Backer et al. 73NatPS243, 77.

28. Hesse $73 A A 27,373$.

29. Hesse et al. $74 A A 31,409$.

30. Udaltsov et al. 74Dokl.Akad.Nauk SSSR, Ser.Mat. Fiz.218, 803; AA37, 21.
31. Taylor $75 A p J 195,513$.

32. Manchester $75 A p J 196,83$.

33. Helfand et al. 75ApV198, 661 .

34. Bruck 73NatPS242, 58.

35. Huguenin et al. 73ApJ181, L139.

36. Huguenin et al. 73BAAS5, 285.

37. Manchester $73 A p J 179, \mathrm{~L} 7$.

38. Argyle $73 A p J 183,973$.

39. Hesse et al. 73NatPS245, 57.

40. Ricket et al. 73BAAS5, 18.

41. Cordes et al. 73BAAS5, 18.

42. Backer et al. 73BAAS5, 18.

43. Schönhardt et al. $73 A L 14,61$.

44. Manchester et al. 73BAAS5, 285.

45. Backer 73ApJ182, 245.

46. Page $73 M N 163,29$.

47. Mclean $73 M N 165,133$.

48. Alekseev 73IVUZR 16, 762.

49. Jones et al. $74 M N 166,39 \mathrm{P}$.

50. Rankin $73 B A A S 5,425$.

51. Rankin et al. 74ApJ188, 609.

52. Smith $74 M N 167,43 \mathrm{P}$.

53. Backer et al. 74ApJ189, 137.

54. Sulejmanova et al. $74 A Z h 51,927$; Soviet Astron. 18, 5.

55. Komesaroff et al. 74Nat252, 210.

56. Rankin et al. 74APJ193, L71.

57. Boriakoff et al. 74AL15, 175.

58. Wolszozan et al. 74AA37, 285.

59. Galt et al. 75AJ80, 311 .

60. Sieber et al. 75AA38, 169.

61. Sieber et al. $75 A A 38,325$.

62. Backer et al. 75APJ197, 481. 
63. Ritching et al. 75Nat 257, 293.

64. Manchester $73 B A A S 5,35$.

65. Rankin et al. 73ApJ181,875.

66. Shishov 73AZh50, 941; Soviet Astron. 17, 5.

67. Backer et al. 73BAAS5, 18.

68. Combe et al. $73 A L 14,59$.

69. Schönhardt 73 NatPS243, 62.

70. Gordon et al. 73AA27, 119.

71. Rickett et al. $73 A p J 185,945$.

72. Backer 73BAAS5, 425.

73. Verschuur 73ApJ183, L9.

74. Galt $74 A A 31,325$.

75. Slee et al. $74 M N 167,31$.

76. Gonzàlez et al. 74AA32, 441.

77. Backer $74 A P J 190,667$.

78. Schönhardt $74 A A 35,13$.

79. Crovisier $74 A A 37,189$.
80. Graham $74 A A 37,405$.

81. Weiler 75Nat 253, 24.

82. Vandenberg 73ApJ180, L27.

83. Crane et al. 73Mit.Res.Lab.

Electronics, Quarterly Prog. Rep.108, 51.

84. Mutel et al. 74ApJ193, 279.

85. Kaplan et al. 73NatPS241, 122.

86. Smith 73Nat 243, 207.

87. Cocke 73ApJ184, 291.

88. Manchester et al. $73 A p J 185,951$.

89. Eastlund et al. $73 B A A S 5,35$.

90. Virtamo et al. 73ApJ182, 935.

91. Zheleznyakov 73Uspeckhi Fiz.Nauk109, 777.

92. Ruderman et al. 75ApJ194, 529.

93. Palmer 75ASS34, 209.

\section{NEUTRAL HYDROGEN, $21 \mathrm{~cm}$ (GALACTIC AND EXTR AGALACTIC) AND RECOMBIN ATION LINES}

\section{(I. V. Gossachinskij, B. Zuckerman)}

Three new general sky surveys with a resolution of up to $2 \mathrm{~km} \mathrm{~s}^{-1}$ have appeared. Practically the entire sky has been observed with an angular resolution of 0.5 , and large regions of the sky with a resolution of $10^{\circ}$. The results come rather slowly due to an extremely large amount of information and slow process of data reduction.

All the papers on the Galaxy during the last three years have shown a keen interest in (a) the very small-scale structure and movement of interstellar gas, and (b) very large-scale phenomena, including the Galactic centre region, large features at high latitudes, and $\mathrm{H} I$ streams in the local system. Some new observations have been made of $\mathrm{H} I$ complexes around clusters, H II regions, SNR, dust clouds, spurs. Very intensive observations of the H I absorption line and of the two-component structure of gas have been made. The deuterium line has been found in the spectrum of SGRA with much greater confidence than any before.

Much progress can be seen in the accumulation of data on the $\mathrm{H} \mathrm{I}$ line in external galaxies.

Some puzzling results are available now on the $\mathrm{H} \mathrm{I}$ absorption line in the direction of quasars. Many $\mathrm{H} \mathrm{I}$ maps of nearby galaxies have been synthesized with a resolution of 0.5 , and integrated properties of about $\mathbf{2 0 0}$ more distant galaxies have been obtained.

\section{REFERENCES}

\section{Colloquia, Meetings and Symposia}

1.1 'Atoms and Molecules in Astrophysics', Proc. 12th Sess. Scottish Univ. Summer School, ed. Carson, Roberts, 1972.

1.2 'Les spectres des astres dans l'infrarouge et les micro-ondes', 17 Coll. Inst. Astrophys. Liège, 1972.

1.3 'Interstellar Matter', Swiss Soc. Astron. Astrophys. Geneva, 1973.

1.4 'Interstellar Dust and Related Topics', IAU Symp. 52, ed. Greenberg, Van de Hulst, 1973, $601 \mathrm{p}$.

1.5 'The Formation and Dynamics of Galaxies', IAU Symp. 58, ed. Shakeshaft, 1974, $441 \mathrm{p}$.

1.6 'Galactic Radio Astronomy', IAU Symp. 60, ed. Kerr, Simonson III, 1974, 668 p.

1.7 'Stars and Milky Way System', Proc. First EAM 2, ed. Mavridis, 1974, 368 p.

1.8 'Galaxies and Relativistic Astrophysics', Proc. First EAM 3, eds. Barbanis, Hadjidemetriou, 1974, 247 p. 
1.9 'The Interstellar Medium', Proc. NATO Adv. Study Inst., ed. Pinka, 1974, 309 p.

1.10 'Molecules in the Galactic Environment', ed. Gordon, Snyder, 1973, 493 p.

\section{Large surveys of $H$ I line}

2.1 Weaver $73 A A$ Sup8, 1.

2.2 Simonson 73AA Sup10, 283.

2.3 Burton 73AA Sup12, 142. res. 10'

2.4 Heiles $74 A A$ Sup14, 127.

2.5 Weaver 74AA Sup17, 1 .

2.6 Weaver $74 A A$ Sup 17, 251.

2.7 Heiles 74AA Sup14, 557.

2.8 Kerr et al. $75 A A$ Sup in press.

2.9 Westerhout et al. $75 A A$ Sup in press.

3. Observations of the hydrogen line in emission

3.0 Rohlts et al. 72AJ77, 711 .

3.1 Garzoli 72Carnegie Publ. Inst. No 629, 123.

3.2 Williams 73AA Sup8, 505. scale $\mathrm{T}_{\mathrm{B}}$

3.3 Pöppel et al. 73AA Sup9, 289.

3.4 Verschuur 73AA24, 35.

3.5 Verschuur 73AJ78, 573 .

3.6 Simonson 74AA Sup16, 447.

3.8 Fejes 74AJ79, 25.

3.9 Simonson et al. 74BAAS6, 436.

3.10 Bystrova et al. 75Izv.SAO7, 70.

3.11 Bystrova et al. 75Izv.SAO7, 87.

3.12 Mirabel 75ASS33, 23.

3.13 Harten et al. 75AJ80, 307.

3.14 Lindblad 74AA Sup16, 207.

4. Hydrogen line and spiral structure

4.0 Davies $72 M N 160,381$. Outer and HVC

4.1 Kerr 73IEEE61, 1182.

4.2 Garzoli et al. 73ASS25, 207.

4.3 Minn et al. 73ASS24, 393.

4.4 Verschuur 73AA27, 73.

4.5 Burton 73PASP85, 679. scale $T_{B}$

4.6 Verschuur $73 A J 78,898$. new map review

4.7 Piddington $73 M N 162,73$. H I/theory

4.8 Burton et al. 74AA33, 425 .

4.9 Weaver 74IAU Symp.60, 573.

4.10 Rohlfs 74AA35, 177.

4.11 Henderson 74BAAS6, 344.

4.12 Georgelin 75CR 280, 13349.
5. The details of spiral arms; hydrogen layer

5.1 Shane $72 A A 16,118$.

5.2 Quiroga 73BAS Argent. No.17, 42.

5.3 Garzoli et al. 73ASS25, 207.

5.4 Yuan et al. 73ApJ185, 453.

5.5 Verschuur 73AA27, 407.

5.6 Verschuur 73AA24, 193.

5.7 Jackson et al. 74ApJ190, 53.

5.8 Wallace 74BAAS6, 345 .

5.9 Quiroga $74 A S S 27,323$.

5.10 Quiroga 75ASS35, 67. (see also 4.6)

6. $H I$ in the Galactic Center

6.1 Kazes et al. 73AA22, 413.

6.2 Sanders et al. 73AA26, 365.

6.3 Wrixon et al. 73AA Sup11, 339.

6.4 Simonson et al. $73 A A 27,337$.

6.5 Sanders et al. $74 \mathrm{Ap} / 188$, 489.

model

6.7 Saraber et al. 74AA36, 365.

6.8 Kaifu et al. 74PASJ26, 117.

6.9 Oort 74IAU Symp.60, 551.

6.10 Cohen 75MN171, 659.

6.11 Peters 75ApJ195, 617. model

6.12 Sandqvist $74 A A 33,413$.

7. $H I$ in or near some galactic objects

7.1 Verschuur 74ApJ Sup27, 283.

spurs

7.2 Fejes et al. $73 A A 25,85 . \quad$ spurs

7.3 Assousa et al. 73BAAS5, 410.

7.4 Assousa et al. $73 A J 78$, 885.

7.5 Isobe 73IAU Symp.52, 433.

SNR

7.6 Tovmassian $73 A J P 26$, 829.

7.7 Tovmassian et al. $73 A J P 26,837$.

7.8 Tovmassian et al. $73 A J P 26,843$.

7.9 Tovmassian et al. $73 A J P 26,853$.

7.10 Tovmassian et al. 73AJP26, 861.

\section{SNR}

association

open cl.

open cl.

open cl.

open cl.

open cl. 
7.11 Knapp et al. $73 A p J 186$, 831.

7.12 Verschuur 73Icarus19, 329.

7.13 Verschuur 73AJ78, 573.

7.14 Sullivan et al. 73AA29, 369.

7.15 Bajaja et al. 73ASS24, 65.

7.16 Simonson 73IAU Symp. 52, 227.

7.17 Myers 74BAAS6, 221.

7.18 Knap et al. 74AA33, 463.

7.19 Sato 74PASJ26, 459.

7.20 Verschuur 74BAAS6, 225.

7.21 Grayzeck et al. 74AJ79, 368.

7.22 Cornett 75AA38, 157.

7.23 De Noyer 75ApJ196, 479.

8. H I in the dust clouds

8.1 Simonson 73AA23, 19.

8.2 Davies 73IAU Symp. 52, 251.

8.3 Knapp 73IAU Symp.52, 243.

8.4 Braunsfurth et al. 73IAU Sy mp.52, 231.

8.5 Seki 73AA28, 207.

8.6 Kerr et al. 74AJP27, 285.

8.7 Minn et al. 74BAAS6,221.

8.8 Heiles 74IAU Symp.60, 625.

8.9 Knapp 74AJ79, 521.

8.10 Knapp $74 A J 79,541$.

8.11 Knapp et al. 74AAp35, 361.

8.12 Gossachinskij et al. $75 A Z h 52,34$.

8.13 Heiles et al.75ApJ199, 361.

9. H I local

9.1 Fejes et al. 73AA24, 1.

9.2 Wesselius et al. 73AA24, 15.

9.3 Wesselius $73 A A 24,35$.

9.4 Lindblad et al. 73AA24, 309.

9.5 Baker 73AA26, 203.

9.6 Elliot et al. 73ASS20, 111.

9.7 Henderson 73AJ78, 381 .

9.8 Takakubo 74IAU Symp.60, 631.

9.9 Heiles 74IAU Symp.60, 625.

9.10 Weaver $74 H A 3,423$.

9.11 Simonson et al. 74BAAS6, 436.

9.12 Burton et al. 74AA34, 75.

9.13 Lindblad $74 H A 3,381$. globular cl.

stars

H II

H II

H II

H II

SNR

SNR

H II

spurs

stars

$\mathrm{X}$-sources

SNR

SNR

review

review

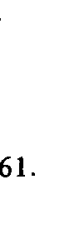

9.14 Lindblad 74IEAM2, 65. (see also 7.2)

10. Cloud structure of $H I$ and intercloud medium

10.1 Baker $73 A A 23,81$.

10.2 Bejsekova et al. 73AZh50, 424.

10.3 Verschuur 73AJ78, 898.

10.4 Baker et al. 73AL13, 199.

10.5 Hachenberg 74IEAM2, 20.

10.6 Baker 74ApJ194, L109.

10.7 Verschuur 74ApJ Sup27, 283.

10.8 Verschuur 74ApJ Sup27, 65.

10.9 Rohlfs 74AA35, 177.

10.10 Schwartz et al. 74IAU Symp.60, 45.

10.11 Hachenberg et al. 74IEAM2, 120.

10.12 Mebold 74AA30, 329.

10.13 Baker et al. 74ApJ187, 223.

10.14 Davies et al. 75MN170, 95.

10.15 Quiroga 75ASS35, 67.

10.16 Baker 75ApJ198, 281. (see also 4.2)

11. High and intermediate velocity clouds

11.1 Elliot et al. 73ASS20, 111.

11.2 Verschuur 73AA22, 139.

11.3 Hulsbosch et al. 73AA22, 153.

11.4 Wesselius $73 A A 24,35$.

11.5 Verschuur $73 A A 27,407$.

11.6 Giovanelli et al. 73AA Sup12, 209.

11.7 Wesselius et al. 73AA24, 15.

11.8 Silk et al. $73 A L 13,143$.

11.9 Takakubo 74IAU Symp.60, 631.

11.10 Davies 74IAU Symp.60, 599.

11.11 Wright $74 A A 31,317$.

11.12 Saraber et al. $74 A A 36,365$.

11.13 Hulsbosh 75AA40, 1.

11.14 Verschuur $75 A R A A 13,257$. (see also 4.0)

12. $H$ I line in absorption

12.1 Kazes et al. 73AA22, 413.

12.2 Dickel $73 A L 15,61$.

12.3 Williams $73 A A 28,309$.

12.4 Greisen 73ApJ184, 363.

12.5 Greisen $73 A p J 184,379$. a.s.

12.6 Gordon $73 A A 27,119$. pulsar

12.7 Gonzalez $73 A L 13,229 . \quad$ pulsar

12.8 Sullivan $73 A A 29,369$. H II

12.9 De Young et al. $73 A p J 185,809 . \quad$ eg

12.10 Brownetal. 73 ApJ184, L7. eg

12.11 Gottesman et al.

12.12 Knapp et al. 74AA33, 463.

12.13 Baker $74 A p J 194$, L109. a.s. 


$\begin{array}{lll}12.14 & \begin{array}{l}\text { Gonsàles } 74 A A 32, \\ 441 .\end{array} & \text { pulsar } \\ 12.15 & \begin{array}{l}\text { Graham } \text { et al. } 74 A A 37, \\ 405 .\end{array} & \text { pulsar } \\ 12.16 & \begin{array}{l}\text { Sato } \text { et al. 74An.Tokyo } \\ \text { AO14, 120. }\end{array} & \text { H II } \\ 12.17 & \begin{array}{l}\text { Wright 74AA31, 283. } \\ \text { Weg }\end{array} \\ 12.18 & \begin{array}{l}\text { Weliachev 74ApJ191, } \\ 639 .\end{array} & \text { eg } \\ 12.18 & \text { Quiroga 75ASS35, 67. } & \\ 12.19 & \text { Gordon 75AA40,27. } & \text { pulsar } \\ 12.20 & \text { Cohen 75MN171,659. } & \text { g.c. } \\ 12.21 & \begin{array}{l}\text { Lasareff } 75 A A 42,25 . \\ \text { see also 7.14, 8.12) }\end{array} & \text { survey } \\ & \text { (see }\end{array}$

13. The temperature of $H I$ regions

13.0 Knapp et al. 72AJ77, 717.

13.1 Baker 73AA23, 81 .

13.2 Hachenberg 74IEAM2, 120. (see also $7.18,10.4,10.10,10.11,10.12$, $10.14,12.13$ )

14. The motions of gas

14.1 Burton 73PASP85, 508 . gal.rot.

14.2 Minn et al. 73AA24, 393. as/other

14.3 Yuan et al. $73 \mathrm{ApJ185}, 453$. ss

14.4 Ariskin $73 S A J 50,83$. ss

14.5 Sofue et al. 74AA36, 237.

14.6 Gossachinskij 75Izv.SAO 7, 96. (see also 4.8, 5.7, 6.4, 7.2, 9.4, 9.10, $9.12,10.1,10.5,10.8,10.9,10.12)$

15. $H I$ in galaxies, surveys and review papers

15.0 Gottesman et al. 73ApJ184, 71.

15.1 Balkowski 73AA29, 43. abs. Qss. correl. param.

15.2 Lewis et al. $73 M N 165$, 213.

29 gal.obs

15.3 Roberts $73 A A 26,483$.

15.4 Kellman et al. $73 A$ pJ184, 753.

rot.curve

15.5 Boting $\mathrm{H}$ I/types 217.

15.6 Gerard 73AA28, 95. halo in gal-s

15.7 Bottinelli et al. 73AA25, 451.

15.8 Lauque et al. $73 A A 23$, 253.

15.9 Balkowski et al. 73AA23, 139.

15.10 Bottinelli et al. 73AA22, 281.

15.11 Peterson et al. 74AJ79, 767.

Markarian

gal-s

peculiar

gal-s
15.12 Knapp et al. 74AJ79, 667.

E gal-s

15.13 Emerson et al. 74IEAM3, 19.

15.14 Davies et al. 74IEAM3, $19 . \quad$ obs.

15.15 Dickel et al. 75AJ80, 584.

15.16 Dean et al. 75MN170, 503.

15.17 Lewis et al. 75MRAS78, vel. H I/opt, $75 . \quad 202$ gal-s

15.18 Bottinelli et al. 75AA41, Markarian 61.

15.19 Balkowski et al. $74 A A 34$, 43.

15.20 Shostak 75ApJ198, 527. Scd-gal.obs.

16. HI in galaxies, single ones

16.0 Brown et al. 73ApJ184, L7.

abs. $3 \mathrm{c} 286$

16.1 Bottinelli et al. 73AA29, 425.

NGC5236

16.2 Guibert 73AA29, 335. M32, NGC205

u.1.

16.3 Huchtmeier 73AA23, 93. NGC4244 Scd

16.4 Davies 73MN161, 25P NGC4151

Seyfert

16.5 Huchtmeier 73AA22, 27. NGC3109 Irr

16.6 Guibert 73AA Sup12, 263. M31

16.7 Wright 73ApJ179, 453. M33 outer part

16.8 Hucht meier $73 A A 22,91$. M33

16.9 Erkes et al. 73BAAS5, 430.

16.10 De Young et al. 73ApJ $185,809$.

SMC

16.11 Guibert $74 A A 30,353$. Per-A

16.12 Madore et al. 74ApJ191, 317.

16.13 Siefert et al. 74BAAS6, 435.

16.14 Garozzy et al. $74 A A 30$, 21.

16.15 Wright 74AA31, 283.

16.16 Davies $75 M N 170,45 \mathrm{P}$

M31

M33

NGC 3359 SBC

compact

gal-s

Cent-A abs.

M31, HVC

17. $H$ I in galaxies, aperture synthesis

17.1 Warner et al. $73 M N 163$, 163.

M33

17.2 Allen et al. 73AA29, 447. M101

17.3 Shostak et al. 73AA24, NGC2403, 405.

4236

17.4 Shostak et al. 73AA24, 411.

compact gal-s
17.5 Weliachev et al. $73 A A 24$, 59.

17.6 Rogstad et al. 73AA22, 111.
M51

NGC6946, IC 342 
17.7 Wright et al. 73AL13,1. Maffei 2

17.8 Seilestad et al. 73ApJ184, IC2574, NGC 343. 7640

17.9 Emerson et al. 73MN165, $9 \mathrm{P}$. M31

17.10 Emerson $74 M N 169,607$. M31

17.11 Rogstad et al. 74ApJ193, 309.

17.12 Allen et al. 74IAU Symp. M101, $58,425$.

NGC5383

17.13 Rots et al. 74AA31, 245.

17.14 Shostak et al. 74AA31, 97.

17.15 Gottesman et al. $75 \mathrm{ApJ}$ $195,23$.

17.16 Weliachev $74 A p J 191$, 639.

M81

IC10

M81

abs. M82

18. Galaxies in groups and clusters, intergalactic medium

18.0 Davies et al. 73MN165, 231.

18.1 Mirabel et al. 73AA22, 437.

18.2 Smart $73 A A 24,171$.

gal.from.

Virgo cl.

tail from

SMC

intergalactie

Coma cl.

18.3 Balkowski et al. 73AA25, Stefan 319.

quintet

18.4 Brown et al. 73ApJ184, L7.

18.5 Gottesman et al. 73ApJ 184, $\mathrm{L} 1$.

abs. $3 \mathrm{C} 286$

18.6 Shostak 74ApJ187, 19.

18.7 Wright et al. $74 A A 36,441$

18.8 Bottinelli et al. 74AA36, 461.

18.9 Mathewson 74IAU Symp. 60, 617.

18.10 Davies 74IAU Symp.58, 119.

18.11 Mathewson et al. 74ApJ 190, 291.

18.12 De Young et al. 74ApJ $189,1$.

18.13 Huchtmeier et al. $75 A A$ 41, 477.

18.14 Winter et al. $75 M N 172$, 1.

abs.QSS

Stefan quintet

u.l.cl.

gal. in Coma

cl.

Mag.stream

out of gal-s

Mag.stream

u.l. in $\mathrm{cl}$.

interacting

gal-s

18.15 Cohen et al. 75MN170, 23P.

18.16 Mathewson et al. 75ApJ 195, L97.

18.17 Field 74IAU Symp.63, 13.

Mag.stream

Sculptor group Intergal. medium, review

19. Recombination H-lines, surveys and review papers

19.1 Simpson 73ASS20, 187 . Stark, survey
19.2 Gordon 74IAU Symp.60, 151.

19.3 Guljaev et al. 74AZh51, 1237.

Ahmad 74ApJ194, 503. theory

19.5 Terzian et al. 74IAU Symp. 60, 241.

19.7 Gull et al. 74ApJ192, 63 . survey

19.8 Gordon et al. 74ApJ192, 337.

19.9 Parrish et al. 75ApJ198, 349.

19.10 Hoangh-Binh 72/see ref. 1.2), 367.

19.11 Pyatunina 73Soviet Astron.17, 5.

19.12 Kerr et al. 74IAU Symp. 60,81 .

survey

survey

line width

$\operatorname{grad} \mathrm{T}$ e $\mathrm{N}_{\mathrm{e}}$

review

19.13 Berulis et al. 75Pisma $A Z h 1,28$.

H56 $\alpha$ H II-5

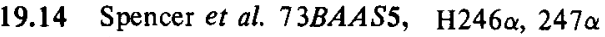
452.

$\mathrm{H} \mathrm{I}-5$

19.15 Parrish et al. 73BAAS5, H226 $248 \alpha$ 452.

H II-3

20. Recombination H-lines in $H I I$ regions

20.0 Parrish et al. 72ApJ178, $\mathrm{H} 221 \alpha, \mathrm{H} 248 \alpha$ 673.

in W51

20.1 Matthews et al. 73MN165, 173.

20.2 Lada et al. $73 A p J 183$,

20.3 Cato. 73 Onsala Obs.

H $166 \alpha$ g.c.

$\mathrm{H} 94 a$

NGC7938

Res. Note, No. 114.

$\mathrm{H} 183 \alpha$, 230ß H II-5

20.4 Terzian $73 A J 78,894 . \quad \mathrm{H} 109_{\alpha} \mathrm{Cyg} \mathrm{X}$

20.5 Pedlar et al. 73MN165, H166 $\alpha$ Ros, 381.

NAm

20.6 Chaisson $73 A p J 186,545 . \quad H 94 \alpha, 148 \delta$

20.7 Brown et al. 73ApJ185, 843. OriA

20.8 Waltmann $73 A p J 185$, L35. H42 $\alpha$ OriA

20.9 Chaisson $73 A p J 182,767$. OriB

20.10 Waltman et al. 73ApJ182, 489.

$\mathrm{H} 66 \alpha$

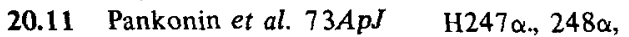
180, L113.

W49A

20.12 Sullivan $73 A A 29,369$. H166 $\alpha 3$

20.13 Lockman et al. $73 A p J 182$, 25.

20.14 Pedlar et al. $74 M N 168$, 577.

H159 $\alpha$ Gal. Cent.

20.15 Macleod et al. 74JRASC $68,266$.

$\mathrm{H} 166 \alpha$ in $\mathrm{H} 2-3$

20.16 Viner et al. 74BAAS6, 349.

$\mathrm{H} 85 \alpha \mathrm{W} 12$

20.17 Pankonin et al. 74AA37, $\mathrm{H} 221 \alpha, 247 \alpha$, 411.

$248 \alpha$, W51, W49 
20.18 Pauls $74 A A 34,327$.

20.19 Huchtmeier $74 A A 32$, 335.

20.20 Balik et al. $74 A p J 188$, 45.

20.21 Gardner et al. 75AL16, 29.

20.22 Gardner et al. $75 M N 171$ 29 P.

20.23 Brown et al. 75ApJ200, L155.

20.24 Churchwell et al. $74 A A$ 32, 283.

20.25 Macleod et al. 75AA42, $\quad \mathrm{H} 85 \alpha$ 195. NGC2024

20.26 Thonnard 73BAAS5, 451. H67 $\alpha$ OriA

20.27 Zeilik et al. 73BAAS5, 451 . H92 $\alpha$ M16

20.28 Zeilik et al. 74AJ79, 786. H92 $\alpha$ M16

20.29 Willson et al. 74BAAS6, 350 .

20.30 Hoangh-Binh et al. $74 A A$ $35,49$.

20.31 Lockman, Brown $75 \mathrm{ApJ}$ 201 , in press.

21. Recombination H-lines in other galactic object

21.1 Bignell $73 A p J 186,889$.

$\mathrm{H} 166 \alpha \mathrm{W} 44$

21.2 Cesarsky et al. $73 A p J 184$, 83. SNR

21.3 Downes $74 A A 34,133$.

21.4 Chaisson $74 A p J 189,69$.

SNR

H1 $34 \alpha$ SNR

SNR

21.5 Terzian et al. 74AJ79,1018. planet.neb

21.6 Bignell $74 A p J 193,687$. planet. neb

21.7 Higgs 74JRASC68, 295 . widths, planet. neb
21.8 Mcgee et al. 74AJP27, 729.

21.9 Pankonin 75AA38, 445 .

$\mathrm{H} 109 \alpha, \mathrm{LMC}$

H248 $\alpha$ SNR

22. Recombination H-lines in $H I$ and dust clouds, and in diffuse medium

22.0 Gordon $72 C I A L 17,409$.

22.1 Gordon 73ApJ184, 77.

22.2 Davies et al. $73 M N 165$, 149.

22.3 Pedlar 73Obs93, 166.

22.4 Lackson et al. 73BAAS5, 331.

22.5 Zuckerman 74IAU Symp. $60,45$.

dark cloud

H166 $\alpha$ diffuse diffuse, review

H110 $\alpha$ diffuse

22.6 Chaisson et al. $74 A \mathrm{PJ} 189$, 227.

H I 190, 35 .

22.8 Jackson et al. 75ApJ196, 723.

H110 $\alpha$ diffuse

23. Recombination lines from heavier elements

23.1 73BAAS5, 451.

23.2 Brown et al. $74 A p J 189$, 253.

dark cloud

23.3 Zuckerman et al. 74ApJ $190,35$.

23.4 Dupree 74ApJ187, 25.

23.5 Chaisson 74ApJ191, 411.

23.6 Knapp et al. 75ApJ196, 167. (see also 20.6, 20.18, 21.3)

23.7 Brown et al.74AA 32, 83. C dark cloud

23.8 Chaisson 73BAAS5, 451. heavy el.

23.9 Dupree $73 B A A S 5,22$. carbon

\section{RADIOASTRONOMY INVESTIGATIONS OF INTERSTELLAR MOLECULES}

\section{(V.K. Khersonsky)}

It is possible to claim that the in terest in the subject mentioned above is continuously increasing. For the last three years 9 new interstellar molecules and some radio lines (which have not yet been identified) have been discovered. The total number of molecules observed in the microwave region is 31 . Most of the interstellar molecules are observed in our Galaxy. However some reports deal with the detection of microwave molecular radiation from extragalactic sources.

The aim of the present review is to give a complete bibliography of papers published in 1973 , 1974 , and partly in 1975.

\section{A. Carbon monoxide (CO) lines}

Carbon monoxide has been studied at the $2.6-\mathrm{mm}$ wavelength $(\mathrm{I}=1-0$ transition) in the following objects: the galactic center $(36,38,214,215,304,305,314,315)$; Sgr B2 (411); the 
Orion nebula $(101,124,146,212,276,362,411,413) ; C y g X$-region (84); in the spectrum of $\rho$ Ophiuchi (102); IC 1396 (223): W49, W51 (312); W3, NGC 7538 (397); M 17 (202); M82, NGC 253 (282); M82, NGC 253, M31, M51 (345), and some other sources (77, 128, 195, $196,239)$. Special studies of radiation have been carried out for optically dark clouds $(18,19$, $225,319)$; dust clouds $(103,240,241)$; the sources associated with $H$ II regions $(90,135,229)$; and the star formation regions (222). General investigation of the distribution of CO molecules in the Galaxy has been made $(309,310,399,400)$. The structure and the detailed maps of some clouds have been studied $(124,146,212,214,215,319)$. The significance of $\mathrm{CO}$ in the cooling of interstellar gas clouds and the collapse of massive clouds has been discussed $(18,19$, $81,134,222,224,228,311,312,355)$. Molecules of ${ }^{12} \mathrm{C}^{17} \mathrm{O}(104)$ and ${ }^{13} \mathrm{C}^{16} \mathrm{O}(135,146$, 202, 223) have been observed. Emission in $I=2 \rightarrow 1$ line of ${ }^{12} \mathrm{C}^{16} \mathrm{O}$ and ${ }^{13} \mathrm{C}^{16} \mathrm{O}$ has been investigated $(135,270)$. Laboratory measurements and calculations of $\mathrm{CO}$ frequencies have been reported (227). The cross-section of excitation of $\mathrm{CO}$ rotational levels in the $\mathrm{CO}-\mathrm{H}_{2}$ collisions has been studied (64).

\section{B. Hydroxyl radical $(\mathrm{OH})$ lines}

General studies of $\mathrm{OH}$ lines have been carried out for the following objects: W3 $(143,155$, 192, 264); W3A (52, 53); W10, W12 (52, 53); W22 (52, 53, 325); W28 (266); W30H (230); W31 (394); W37 (409); W41 (140, 141); W43 (140, 141, 322, 409); W49 (155); W49A, W49B (265); W49N (230); W51 (100, 140, 141, 323, 324, 395); W51A (52, 53); W73B (99); W75 (408); W75B (299); NGC 2068 (169, 170); NGC $2071(169,170)$; NGC $2438(145,409)$; NGC 4945 (389); NGC 6334 (264, 327); NGC 6334N (192); the galactic center $(206,306)$; SgrB2 (155); Orion A (267); the Orion nebula (413); ON-3 (409); V 1057 Cyg (217, 218, 219); Cyg X-region (263); VY CMa (264); NML Cyg (8); 3C 353 (140, 141); Southern Coalsack (32); the Carina nebula (85); M20 (328), and some other sources $(5,26,27,50,51,89$, $160,166,173,184,185,209,286,287,303,367,369,402)$. Investigation of radiation from optically dark and dust clouds $(66,67,68,190,237,256,257,393)$, as well as from IR sources $(48,398,403)$ has been carried out. Emission and absorption of excited rotational levels have been observed $(15,121,192,261,283,284,294,299)$. Some anomalies have been discussed $(367,389,390)$. Observations of $\mathrm{OH}$ maser sources have been reported $(40,75,150,189,204$, $205,207,230,233,357,404)$. Interferometry and VLBI measurements of OH sources have been made $(76,155,191,192,232,279,406)$. The structure and the detailed distribution of the gas in the clouds have also been investigated $(65,143,144,189,216,217,218,219,220$, $266,303,324)$. Models for the $\mathrm{OH}$ masers have been proposed $(41,42,43,45,46,47,63,78$, $149,182,183,203,210,261,278,326,380$ ). Polarization of $\mathrm{OH}$ emission has been studied $(151,217,218,219)$. Zeeman splitting of spectral lines has been used to determine the magnetic field in the molecular clouds (75). Calculations of frequencies and probabilities of radio transition of OD have been performed (186); the estimate of the OD line strength towards the galactic center can be found in (6). The frequencies of the ${ }^{17} \mathrm{OH}$ lines have been reported (376).

\section{Water in $\left(\mathrm{H}_{2} \mathrm{O}\right)$ lines}

$\mathrm{H}_{2} \mathrm{O}$ emission has been observed in the following objects: W3, W49, W51 (353); W37, W43 (409); W75 (353, 408); Sgr B2 (353, 378); the Orion nebula $(353,413)$; VYCMa, ON1 (353); ON3 (409); NGC 2438 (409); NGC 6334 (353); far-infrared objects (387); OH sources (407) and some other sources $(17,59,142)$. Emission lines of the regions associated with $\mathrm{H}$ II regions and IR stars have been studied $(11,48,49,88,220,221)$. Observations of $\mathrm{H}_{2} \mathrm{O}$ masers have been reported $(91,180,181,308,357)$. In terferometry and VLBI measurements of $\mathrm{H}_{2} \mathrm{O}$ emission features have been made $(16,20,191,193,200,255,262,280)$. The sizes and the relative positions of some $\mathrm{H}_{2} \mathrm{O}$ sources have also been determined $(189,230)$. The nature and models of $\mathrm{H}_{2} \mathrm{O}$ masers have been discussed $(43,45,78,79,80,210,226,344)$. Polarization of emission has been studied (24). Detection of HDO in Orion A has been carried out $(373,374)$. (See also (275)). Laboratory measurements and calculations of frequencies and line strengths of $\mathrm{H}_{2} \mathrm{O}$ have been reported (82). 


\section{Hydrogen cyanide (HCN) lines}

There have been general studies of the HCN lines in the following objects: Sgr B2 (178); Orion nebula $(124,146,178,263)$; IC $1396(223)$, in the direction of M8 (126) and M17 (202) and some other sources $(58,59,195,196,250,251)$. The papers $(58,59,199,411,413)$ deal with the interpretation of observations. The structure of the emission regions in the Orion nebula has been discussed $(124,146) . \mathrm{H}^{13} \mathrm{C}^{14} \mathrm{~N}$ has been observed in the Orion nebula (254) as well as DCN $(347,401)$. Collisional excitation of the rotational levels of $\mathrm{HCN}$ by $\mathrm{H}_{2}$ has been studied (147).

\section{E. Formaldehyde $\left(\mathrm{H}_{2} \mathrm{CO}\right)$ lines}

Observations of emission and absorption of different rotational $\mathrm{H}_{2} \mathrm{CO}$ lines have been made for the following objects: the galactic center $(306,313)$; SgrA $(235,388,392)$; Sgr B2 (289), the Orion nebula $(86,105,107,146,156,413,425)$, W3 $(245,391)$; W31 (394); W49, W51 (312); NGC 2264 (244); NGC 7538 (95) CasA (360); in the direction of M17 (201, 202); Cen A (259), the Carinal nebula (119); Southern Coalsack (31); Cyg X-3 (243) and some other sources $(111,136,158,159,160,166,369,396)$. Observations for para- $\mathrm{H}_{2} \mathrm{CO}$ have been reported $(4,176,177)$. (See also $(248)$ ). Investigations of $\mathrm{H}_{2} \mathrm{CO}$ radiation have been carried out in optically dark clouds $(106,244,245,246,247,393)$, dust clouds $(69,87,103,160$, $256,257,354)$ the regions associated with $\mathrm{H}$ II regions $(229,410)$. Interferometry measurements of formaldehyde line sources have been made $(111,112)$. Detailed maps and the structure of such clouds have also been discussed $(107,146,354)$. Anomalies of $\mathrm{H}_{2} \mathrm{CO}$ lines have been studied $(69,103,160,273,357,412) . \mathrm{H}_{2}{ }^{13} \mathrm{C}^{16} \mathrm{O}$ has been observed $(410) . \mathrm{H}_{2} \mathrm{CO}$ formation mechanisms have been discussed $(73,242)$.

\section{F. Other molecules}

$\mathrm{CH}$ radical. The molecule of $\mathrm{CH}$ has been observed $(288,295,296,297,298,300,301,371$, $372,416)$. $\mathrm{CH}$ radio line formation mechanisms have been discussed $(44,120)$. The problem of formation of $\mathrm{CH}$ and its abundances have been studied $(10,21,23,98,194,331,340,341$, 384).

Silicon monoxide ( $\mathrm{SiO})$. SiO observations have been reported $(249,348)$. Interpretation of the observations can be found in $(134,272,413)$. Maser emission sources have been investigated $(39,171,172,175,334,358)$. A model for explanation of pumping of molecular levels has been proposed (198). Precise laboratory measurements and calculations of SiO frequencies have been made (227).

Carbon monosulphide (CS). CS radiation has been observed $(146,195,196,213,223,250$, 251,312 ). The emission mechanism and the significance of this molecule in cooling of dense molecular clouds have been studied $(134,228,311,312,413)$. Observations of ${ }^{12} \mathrm{C}^{33} \mathrm{~S}$ and ${ }^{12} \mathrm{C}^{34} \mathrm{~S}$ have been reported (375). Frequencies of radio transitions of all isotopic species have been measured in the laboratory (227).

Sulphur monoxide (SO). SO observations have been made in Sgr B2 (178), Ori A $(60,61$, 178), IC 1396 (223), and in the direction of M17 (202). Unsuccessful searches for SO in W51, IRC + 10216 and Heiles cloud 4 have been reported (4). Observations have been interpreted and determination of the magnetic field by measurements of Zeeman splitting of the rotational levels of $S O$ in Orion $A$ has been carried out $(60,61,413)$. The SO formation mechanism has been discussed (260). The frequencies of the SO radio transitions have been measured in the laboratory (359).

Carbonil sulphide (OCS). OCS lines have been observed in Sgr B2 $(4,346)$. Unsuccessful searches for OCS have been carried out (4) in W51, IRC + 10216 and Heiles cloud 4. Laboratory measurements and calculations of OCS line frequencies have been made (231). The OCS formation mechanisms have been discussed (260).

Ammonia $\left(\mathrm{NH}_{3}\right)$. This molecule has been observed in Orion A $(253,254)$, W3, W43, W51, 
Dr-21 (253), in cool and dark clouds (56), and in the direction of Hat Greek (55). The laboratory studies of $\mathrm{NH}_{3}$ microwave spectra have been carried out (277).

Thioformaldehyde $\left(\mathrm{H}_{2} \mathrm{CS}\right)$. The $\mathrm{H}_{2} \mathrm{CS}$ microwave line has been investigated in Sgr B2 (92, 93, 113, 321). Formation reactions have been discussed (260).

Cyanoacetylen $\left(\mathrm{HC}_{3} \mathrm{~N}\right)$. Observations of $\mathrm{HC}_{3} \mathrm{~N}$ radiation in $\mathrm{Sgr} \mathrm{B} 2$ have been made (122, $236,252,346)$. Results of unsuccessful searches for this molecule in W51, IRC +10216 , and Heiles cloud 4 have been reported (4).

Methanol $\left(\mathrm{CH}_{3} \mathrm{OH}\right)$. Studies of $\mathrm{CH}_{3} \mathrm{OH}$ lines in Sgr A, Sgr B2 $(197,285)$, Orion A $(12,13)$ and in the direction of Hat Greek (55) have been carried out. Observations and discussions of anomalous emission have been reported $(12,13,164,208,271)$. Laboratory measurements of ${ }^{12} \mathrm{CH}_{3} \mathrm{OH}$ and ${ }^{13} \mathrm{CH}_{3} \mathrm{OH}$ frequencies have been made $(117,154)$.

Methylamine $\left(\mathrm{CH}_{3} \mathrm{NH}_{2}\right)$. Observations of $\mathrm{CH}_{3} \mathrm{NH}_{2}$ in $\mathrm{Sgr} \mathrm{B} 2$ and Orion A have been reported $(116,178,179)$. Results of a search for this molecule are also presented in (3). Unsuccessful observations in W51, IRC +10216 and Heiles cloud 4 have been made (4). Anomalies of $\mathrm{CH}_{3} \mathrm{NH}_{2}$ emission in Sgr B2 and Orion $\mathrm{A}$ have been discussed $(178,179)$.

There are also reports on the observations of the following molecules:

Cyanogen $(C N)$ in the Orion nebula $(274,368,413)$;

Silicon sulphide (SiS) in IRC + $10216(249)$;

Hydrogen sulphide $\left(\mathrm{H}_{2} \mathrm{~S}\right)$ in the Orion nebula (413);

Sulphur dioxide $\left(\mathrm{SO}_{2}\right)$ in $\mathrm{Sgr} \mathrm{B} 2(336)$;

Ethynyl radical $\left(\mathrm{C}_{2} \mathrm{H}\right)$ in $\mathrm{Sgr} \mathrm{A}, \mathrm{Sgr} \mathrm{B} 2$, Orion $\mathrm{A}$, and soon $(361,363)$

Isocyanid acid $(H N C O)$ in Sgr B2 $(165,333,346)$;

Formic acid (HCOOH) in Sgr B2 (405);

Methanimine $\left(\mathrm{H}_{2} \mathrm{CNH}\right)$ in $\mathrm{Sgr} \mathrm{B} 2(129)$;

Acetaldehyde $\left(\mathrm{CH}_{3} \mathrm{CHO}\right)$ in $\mathrm{Sgr} \mathrm{B} 2(115)$;

Formamide $\left(\mathrm{HCONH}_{2}\right)$ in Sgr A (139), Sgr B2 $(281,139,291)$;

Methylcyanide $\left(\mathrm{CH}_{3} \mathrm{CN}\right)$ in $\mathrm{Sgr} \mathrm{B} 2(346)$;

Vinilcyanide $\left(\mathrm{H}_{2} \mathrm{CCHCN}\right)$ in Sgr B2 (123);

Methyl acetylene $\left(\mathrm{CH}_{3} \mathrm{C}_{2} \mathrm{H}\right)$ in $\mathrm{Sgr} \mathrm{B} 2$ (333);

Methyl format $\left(\mathrm{HCOOCH}_{3}\right)$ in $\mathrm{Sgr} \mathrm{B} 2(34)$;

Dimethyl ether $\left.\left(\mathrm{CH}_{3}\right)_{2} \mathrm{O}\right)$ in Orion nebula (335);

Trans-ethyl alcohol $\left(\mathrm{CH}_{3} \mathrm{CH}_{2} \mathrm{OH}\right)$ in $\mathrm{Sgr} \mathrm{B} 2(417,418)$.

\section{G. Unidentified lines}

Some unidentified lines have been observed: U81,5 (346); U86,2 (332, 337); U89,2 (37, 253); U90,7 (253); U93,2 (366). Possibilities of identification of these lines have been proposed: (226); (226); (37, 226, 379); (226); (147, 366). See also "Quarterly Summary" on Unidentified Lines.

\section{H. Candidates for radio observations}

Unsuccessful searches have been made for the following molecules: PN (253); nitroxyl HNO (114); thioisocyanid acid $\mathrm{HNCS}(9)$; formil $\mathrm{HCO}$, pyridine $\mathrm{C}_{5} \mathrm{H}_{5} \mathrm{~N}$, acroleine $\mathrm{CH}_{2} \mathrm{CH} \mathrm{CHO} \mathrm{(14);}$ nitric acid $\mathrm{HNO}_{3}$, fulven $\mathrm{C}_{6} \mathrm{H}_{6}(125)$; pyridine $\mathrm{C}_{5} \mathrm{H}_{5} \mathrm{~N}$, pyrimidine $\mathrm{C}_{4} \mathrm{H}_{4} \mathrm{~N}_{2}$ (320); benzonitrile $\mathrm{C}_{6} \mathrm{H}_{5} \mathrm{CN}$, benzaldehyde $\mathrm{C}_{7} \mathrm{H}_{6} \mathrm{O}$, toluene $\mathrm{C}_{6} \mathrm{H}_{5} \mathrm{CH}_{3}$, nitrobenzene $\mathrm{C}_{6} \mathrm{H}_{5} \mathrm{NO}_{2}$, cyclobutanone $\mathrm{C}_{4} \mathrm{H}_{6} \mathrm{O}$ (109). See also the 'Quarterly Summary' I of Negative Searches. The laboratory measurements and calculations of the radio frequencies of $\mathrm{H}_{2} \mathrm{D}^{+}(258), \mathrm{HCO}^{+}(163), \mathrm{HNC}(268)$, $\mathrm{H}_{2} \mathrm{CN}^{+}$(269), $\mathrm{KOH}, \mathrm{KOD}$ (270), $\mathrm{O}_{2}$ (299), pyrrol $\mathrm{C}_{4} \mathrm{H}_{5} \mathrm{~N}$ (25), methylenimine (188), vinilcyclopropan $\mathrm{C}_{3} \mathrm{H}_{6} \mathrm{CHCH}_{2}$ (62) have been reported. Radio intensities of the excited $\mathrm{H}_{2}$ have been calculated (293). Theoretical estimates for the intestellar $\mathrm{H}_{2}$ and $\mathrm{HD}$ rotational lines have been made (96). The $\mathrm{H}_{2}$ formation mechanisms have been discussed $(22,342)$, as well as those for $\mathrm{HD}(108,382)$. Abundance data for $\mathrm{H}_{2}$ estimated from theoretical studies, UV and IR measurements have been reported $(329,333,338,350,351)$. Possibilities of $\mathrm{H}_{2}$ and $\mathrm{N}_{2}$ radio observations in molecular complexes $\mathrm{H}_{2}-\mathrm{NH}_{3}$ and $\mathrm{N}_{2}-\mathrm{NH}_{3}$ have been discussed (187). Radio 
lines of the molecule of $\mathrm{HCl}$ and formation mechanisms of this molecule have been studied (70, 174).

\section{Miscellaneous}

General problems related to interstellar masers have been discussed $(97,130,131,132,133$, $211,290,343)$. Distribution and excitation of molecules in different interstellar objects have been considered $(83,127,137,157,364,365,370,386)$. Molecular formation and destruction processes have been studied $(1,2,7,29,33,54,71,72,74,94,108,110,152,153,162,168$, $238,302,316,317,318,356,381,382,385)$.

\section{REFERENCES}

1. Aannestad 73ApJ Sup25, 205.

2. Aannestad 73ApJ Sup25, 223.

3. Akabane et al. 74Tokyo Astron.Bull. 2nd Ser. 229, 2639.

4. Akabane et al. 74PASJ26, 1.

5. Alksnis et al. 73Astron Tsirk. 794, 7.

6. Allen et al. 74ApJ188, 33.

7. Allen et al. $75 A \mathrm{pJ} 195,81$.

8. Andersson et al. $74 A A 30,475$.

9. Andrew et al. $74 A A 35,295$.

10. Bardsley et al. 73ApJ183, L135.

11. Barret et al. 73Mit Res.Lab.Electronics, Quarterly Progr. Rep. 108, 53.

12. Barret et al. 75ApJ198, L119.

13. Barret et al. 74BAAS6, 341.

14. Batchelor et al. 73AJP26, 557.

15. Baudry $74 A A 33,381$.

16. Baudry et al. $74 A A 36,217$.

17. Baudry et al. $74 A A 31,471$.

18. Berder et al. 73BAAS5, 21.

19. Berder et al. 73ApJ180, L43.

20. Berk et al. 73IVUZR16, 799.

21. Black et al. $73 A L 15,79$.

22. Black et al. $73 B A A S 5,380$.

23. Black et al. $75 A \mathrm{pJ} 199,633$.

24. Bolodna et al. $75 A p J 199,86$.

25. Bolton et al. 74AJP27, 143.

26. Bowers et al. 74AL15, 181.

27. Bowers et al. 74BAAS6, 426.

28. Breuer 73IAU Symp.52, 399.

29. Breuer 73 Umschau 73 Jahrgang, 568.

30. Brooks et al. 74ApJ194, 513,

31. Brooks et al. 74PASA2, 250.

32. Brooks et al. 75MN172, 3.

33. Brown 74IAU Symp.60, 129.

34. Brown 75ApJ197, L29.

35. Brzozowski et al. 74ApJ193, 741.

36. Buhl et al. 73BAAS5, 421.

37. Buhl et al. 73ApJ180, 791 .

38. Buhl et al. 73BAAS5, 32.

39. Buhl et al. 74ApJ192, L97.

40. Burke 74IAU Symp.60, 267.

41. Burdjuzha $74 A Z h 51,26$,

42. Burdjuzha $74 A L 15,189$.

43. Burdjuzha et al. $75 A A 40,233$.
44. Burdjuzha et al. 75Space Res.Inst.Akad. Nauk SSSR Repr. 236.

45. Burdjuzha et al. $74 A Z h 51,346$.

46. Burdjuzha et al. 74AZh51, 669 .

47. Burdjuzha et al. 73AZh50, 481.

48. Caswell 74IAU Symp.60, 423.

49. Caswell et al. 74AJP27, 417.

50. Caswell et al. 74AJP27, 629.

51. Caswell et al. 74AJP27, 597.

52. Chaisson 73BAAS5, 22.

53. Chaisson $74 A J 79,555$.

54. Chapelle et al. 73IAU Symp. 52, 387.

55. Cheung et al. 73BAAS5, 422.

56. Cheung et al. 73ApJ186, L73 .

57. Clark 73BAAS6, 263.

58. Clark et al. 73BAAS5, 332.

59. Clark et al. 74ApJ190, 545.

60. Clark et al. 74BAAS6, 336.

61. Clark et al. $74 A \mathrm{pJ} 191, \mathrm{~L} 87$.

62. Codding et al. 74J.Mol.Spectr.49, 226.

63. Cohen et al. 73ApJ15, 151.

64. Compaan et al. 73ApJ185, L105.

65. Cook $75 M N 171,605$.

66. Crutcher $73 A p J 185,857$.

67. Crutcher $73 B A A S 5,333$.

68. Crutcher $73 A L 14,147$.

69. Crutcher $73 A p J 182, \mathrm{~L} 71$.

70. Dalgarno et al. 74ApJ192, L37.

71. Dalgarno et al. 73ApJ181, 95 .

72. Dalgarno et al. 74ApJ192, 597.

73. Dalgarno et al. 73NatPS245, 100.

74. Dauvillier 73Bull.Soc.Roy.Sci.Liège,42, 413.

75. Davies 74IAU Symp.60, 275.

76. Davies 73IAU Symp. 52, 423.

77. Davies et al. 73AL15, 43.

78. Deguchi 74PASJ26, 437.

79. Dejong 73BAAS5, 23.

80. Dejong 73AA26, 297.

81. Dejong et al. 75ApJ199, 69.

82. Delucia et al. 74J.Phys.Chem.Ref.Data3, 211.

83. Dickel 73IAU Symp.52, 277.

84. Dickel et al. 74BAAS6, 221.

85. Dickel et al. $74 A A 31,5$. 
86. Dieter $75 A p J 199,289$.

87. Dieter $73 A p J 183,449$.

88. Dickinson et al. $73 A p J 180,831$.

89. Dickinson et al. $74 A J 79,938$.

90. Dickinson et al. 74ApJ192, 347.

91. Dickinson et al. 74IRASC68, 265.

92. Doherty et al. 74JRASC68, 265.

93. Doherty et al. 74ApJ192, L157.

94. Donn et al. 74BAAS6, 221.

95. Downes et al. 74ApJ191, L77.

96. Drapatz et al. 74AA36, 211.

97. Dy mamis 73 Nederlands Tijdschr. Natuurkunde39, 193.

98. Elander et al. $73 \mathrm{ApJ184}, 663$.

99. Ellder 73Res.Lab.Electronics, Onsala Space Obs.Chalmers Univ.Techn., Gothenburg, Sweden, Res. Rep.116, 11.

100. Ellder et al. 73Res. Lab.Electronics, Onsala Space Obs., Chalmers Univ.Techn.,Gothenburg, Sweden, Res. Rep. 117, 34.

101. Elliott et al. 73NatPS224, 69.

102. Encrenaz 74ApJ189, L135.

103. Encrenaz et al. 73ApJ184, 361.

104. Encrenaz et al. 73ApJ186, L77.

105. Evans et al. 73BAAS5, 421.

106. Evans et al. 75ApJ196, 433.

107. Evans et al. 75ApJ199, 383.

108. Fehsenfeld et al. 73ApJ183, L25.

109. Fertel et al. 75AL16, 61 .

110. Floyd et al. 73RASC67, 299.

111. Fomalont et al. $72 C I A L 17,453$.

112. Fomalont et al. 73ApJ181, 781.

113. Fourikis et al. 74PASA2, 252.

114. Fourikis et al. $74 A p J 194,41$.

115. Fourikis et al. 74AJP27, 425.

116. Fourikis et al. $74 A p J 191, \mathrm{~L} 139$.

117. Gaines et al. 74ApJ191, L99.

118. Gammon et al. 73BAAS5, 23.

119. Gardner et al. $73 A A 23,51$.

120. Gardner 74PASA 2, 253,

121. Gardner et al. 75MN172, 9p.

122. Gardner et al. 75ApJ197, L73.

123. Gardner et al. 75ApJ195, L127.

124. Gerola et al. 75ApJ196, 473.

125. Giguere et al. 73ApJ182, 477.

126. Giguere et al. $73 A p J 182, \mathrm{~L} 11$.

127. Glassgold et al. 74ApJ193, 73.

128. Glassgold et al. 75ApJ197, 347.

129. Godfrey et al. 73AL13,119.

130. Goldreich et al. 73ApJ179, 111.

131. Goldreich et al. 73ApJ182, 55.

132. Goldreich et al. 74ApJ190, 27.

133. Goldreich et al. 74ApJ191, 93.

134. Goldreich et al. 74ApJ189. 444.

135. Goldsmith et al. 75ApJ196, L39.

136. Gordon et al. 73ApJ182, 41.

137. Gordon et al. 73J.Brit.Interplanet.Soc. 26, 639.

138. Gottlieb et al. 73ApJ184, L59.

139. Gottlieb et al. 73ApJ182, 699 .

140. Goss et al. $73 A A 28,89$.
141. Goss et al. 74IAU Symp.60, 135.

142. Sancisi et al. $74 A A p 35,442$.

143. Goss et al. 75AA40, 439.

144. Goss et al. 73ApJ183, 843.

145. Goss et al. $73 A A 29,435$.

146. Goudis $75 A S S 35,409$.

147. Green et al. 74ApJ193, L89.

148. Green et al. 74ApJ191, 653.

149. Gwinn et al. 73ApJ179, 789.

150. Habind et al. $74 A A 35,1$.

151. Hall et al. $73 M N 162,97$.

152. Harteck et al. 73IAU Symp.52, 395.

153. Harteck et al. 74Ber.Bunsenges.Phys.Chem. $78,168$.

154. Haque et al. $74 A p J 187, \mathrm{~L} 15$.

155. Harvey et al. $74 M N 169,545$.

156. Harvey et al. $74 \mathrm{ApJ189,} \mathrm{L87}$.

157. Heiles $73 A p J 179, \mathrm{~L} 17$.

158. Heiles $73 A p J 183,441$.

159. Heiles et al. 73IAU Symp.52,375.

160. Heiles et al. 75ApJ199, 361 .

161. Heiles $73 A p J 182, \mathrm{~L} 121$.

162. Herbst et al. 73ApJ185,505.

163. Herbst et al. 74ApJ188, 255.

164. Hills et al. 75AA39, 149.

165. Hockind et al. 74ApJ187, L89.

166. Höglund et al. $74 A A 33,389$.

167. Hyland 74IAU Symp.60, 439.

168. Iguchi et al. 73Chem Lett(Japan)5, 505.

169. Johansson et al. $74 A \mathrm{pJ} 189,455$.

170. Johansson et al. 74IAU Symp.60, 301.

171. Johnson et al. 74BAAS6, 452.

172. Johnson 75ApJ197, L69.

173. Johnson et al. 73ApJ182, 67.

174. Jura $74 A p J 190, \mathrm{~L} 33$.

175. Kaifu et al. 75ApJ195, L359.

176. Kaifu et al. 75ApJ196, 719.

177. Kaifu et al. 74IAU Symp. 60, 233.

178. Kaifu et al. 74ApJ191, L135.

179. Kaifu et al. 75ApJ198, L85.

180. Kaufmann et al. 74IAU Symp.60, 293.

181. Kaufmann et al. 74AJ79, 933.

182. Keeley $74 A p J 192,601$.

183. Kegel $74 A A 32,227$.

184. Kerr et al. $74 A A 36,225$,

185. Kerr et al. 74IAU Symp.60, 459.

186. Khersonsky $73 A Z h 50,646$.

187. Khersonsky $74 A Z h 51,1298$.

188. Kirchhoff et al. 73J.Phys.Chem.Ref.Data2, 1.

189. Klochan et al. $74 A Z h 51,354$.

190. Knapp et al. 73AJ78, 453.

191. Knowles 74J.Opt.Soc.Amer.64. 522.

192. Knowles et al. 73ApJ180, L117.

193. Knowles et al. 74AJ79, 925.

194. Krauss et al. 73ApJ183, L139.

195. Kutner 75ApJ199, 79.

196. Kutner et al. 74BAAS6, 341.

197. Kutner et al. 73ApJ183, L27.

198. Kwan et al. 74ApJ194, L97. 
199. Kwan et al. 75ApJ195, L85.

200. Kwan et al. 74ApJ 194, 293.

201. Lada et al. $75 A$ pJ195, 367.

202. Lada et al. 74ApJ189, L35.

203. Lang et al. 73ApJ180, 647.

204. Lekht $74 A Z h 51,341$.

205. Lekht $74 A Z h 51,1113$.

206. Lekht et al. 74AZh51, 681 .

207. Lekht et al. 75Pisma AZh 1, 29.

208. Lees $73 A p J 184,763$.

209. Lepine et al. $74 A A 36,469$.

210. Litvak $73 A p J 182,711$.

211. Litvak $74 A R A A 12,97$.

212. Linke et al. 74ApJ193, L41.

213. Liszt et al. $75 A \mathrm{pJ} 196,709$.

214. Liszt et al. 75ApJ198, 537.

215. Liszt et al. 74BAAS6, 435 .

216. Little $73 A L 13,115$.

217. Lo et al. 74BAAS6, 220.

218. Lo et al. $74 A p J 190, \mathrm{~L} 125$.

219. Lo et al. 73ApJ185, L71.

220. Lo et al. $73 A A 26,487$.

221. Lo et al. 74BAAS6, 437.

222. Loren et al. 73BAAS5, 420 .

223. Loren et al. 75ApJ195, 75.

224. Loren et al. 74ApJ194, L103.

225. Loren et al. 73ApJ185, L67.

226. Lovas $74 A p J 193,265$.

227. Lovas et al. 74J.Phys.Chem.Ref.Data3, 245.

228. Lucas $74 A A 36,465$.

229. Lucas et al. $75 A A 41,233$.

230. Mader et al. 74BAAS6, 442.

231. Maki 74J.Phys.Chem.Ref.Data3, 221.

232. Masheder et al. $74 M N 166,561$.

233. Matthews et al. $73 A A 29,309$.

234. Mayer et al. $73 A p J 182$, L65.

235. McCutcheon et al. 74Nat251, 123.

236. McGee et al. $73 A L 13,25$.

237. Menon 74IAU Symp.60, 299.

238. Metz 73Sci.182, 466.

239. Milman 74ApJ193, L93.

240. Milman et al. $75 A J 80,93$.

241. Milman et al. 75AJ80, 101.

242. Millar et al. $75 M N 170,51 \mathrm{P}$,

243. Minn $75 A A 39,303$.

244. Minn et al. 74BAAS6, 221.

245. Minn et al. 73AL13, 39.

246. Minn et al. 73AA22, 13.

247. Minn et al. 73IAU Symp.52, 269.

248. Morimoto et al. 72 CIAL17, 467.

249. Morris et al. 75ApJ199, L47.

250. Morris et al. 74ApJ191, 349.

251. Morris et al. 71BAAS5, 330.

252. Morris et al. $73 L A U$ Symp. 52, 381.

253. Morris et al. 73ApJ186, 501.

254. Morris et al. 74ApJ192, L27.

255. Moran et al. 74BAAS6, 436.

256. Myers $73 A p J 26,83$.

257. Myers $74 B A A S 6,263$.

258. Novick et al. $73 A$ pJ183, L131.
259. Oconnell et al. $75 M N 170,29 \mathrm{p}$.

260. Oppenheimer et al. 74ApJ187, 231.

261. Palmer 74IAU Symp.60, 191.

262. Papadopoulos 74Stars and the Milky Way System IEAM, 129.

263. Pashchenko $73 A Z h 50,685$.

264. Pashchenko et al. 73IVUZR 16, 1344.

265. Pashchenko et al. 73AA26, 349.

266. Pashchenko et al. 74AA35, 153.

267. Pataki et al. 74BAAS6, 340.

268. Pearson et al. 73ApJ184, L19.

269. Pearson et al. 74ApJ192, 33.

270. Pearson 73J.Chem.Phys. 58, 826.

271. Pellind 75MN172, 41 .

272. Pellind $75 M N 172,421$.

273. Pellind et al. $75 M N 171,103$.

274. Penziaz et al. 74PRL32,701.

275. Phillips et al. $73 A L 15,17$.

276. Phillips et al. 73ApJ186, L19.

277. Poynter et al. 75ApJ Sup29, 87.

278. Pustilnik 74Izv. SAO 6, 16.

279. Reid et al. 75ApJ196, L35.

280. Reisz et al. 73ApJ186, 537.

281. Ribes et al. 73AJP26, 79.

282. Rickard et al. 75ApJ199, L75.

283. Rickard et al. 73BAAS5, 331.

284. Rickard et al. 74BAAS6, 340.

285. Robinson et al. 74AJP27, 865 .

286. Robinson et al. $72 C I A L 17,475$.

287. Robinson et al. 74AJP27, 575.

288. Robinson et al. 74Nat 248, 31 .

289. Rogstad et al. 74AA36, 253.

290. Rosen $74 A p J 190, \mathrm{~L} 73$.

291. Rubin et al. 72CIAL17, 471.

292. Rudnitskij $74 A Z h 51,329$.

293. Ruzmajkina $73 A Z h 50,934$.

294. Rydbeck et al. 73BAAS5, 477.

295. Rydbeck et al. 73Nat 246, 466.

296. Rydbeck et al. 74BAAS6, 222.

297. Rydbeck et al. 73Res.Lab.Electronics, Onsala Space Obs, Chalmers Univ.Techn., Gothenburg, Sweden, Res.Rep. 119, 20.

298. Rydbeck et al. 74BAAS6, 261.

299. Rydbeck et al. 72CIAL17, 507.

300. Rydbeck et al. $74 A A 33,315$.

301. Rydbeck et al. $74 A A 34,479$.

302. Salpeter et al. 73IAU Symp.52, 363.

303. Sancisi et al. 74AA35, 445 .

304. Sanders et al. 73BAAS5, 384.

305. Sanders et al. 74ApJ33, 9.

306. Sandquist 74Stars and the Milky Way System IEAM, 157.

307. Schiff et al. 74ApJ191, L49.

308. Schwartz et al. 74ApJ187, 491.

309. Schwartz et al. 73BAAS5, 21.

310. Schwartz et al. 73ApJ186, 529.

311. Scovill et al. 74ApJ187, L67.

312. Scovill et al. 73ApJ180, 31.

313. Scovill et al. 73ApJ180, 55.

314. Scovill et al. 75ApJ199, L105.

315. Scovill et al. 74ApJ187, L63. 
316. Shimizu 73IAU Symp.52, 405.

317. Shimizu 73Progr.theor.Phys.49, 153.

318. Shimizu 73Viva Origino $2,1$.

319. Simon 74BAAS6, 335.

320. Simon et al. 73ApJ184, 757.

321. Sinclair et al. $73 A J P 26,85$.

322. Slysh 73Astron. Tsirk.798, 1.

323. Slysh $74 A Z h 51,470$.

324. Slysh $74 A Z h 51,685$.

325. Slysh $74 A Z h 51,912$.

326. Slysh $73 A A 14,213$.

327. Slysh 75AZh52, 26.

328. Slysh 75Pisma AZh1, 12.

329. Smith 73ApJ179, L11.

330. Smith 73BAAS5, 32.

331. Smith et al. $73 A p J 183,69$.

332. Snyder et al. 74ApJ189, L31.

333. Snyder et al. 73NatPS243, 45.

334. Snyder et al. 75ApJ197, 329.

335. Snyder et al. 74ApJ191, L79.

336. Snyder et al. 75ApJ198, L81.

337. Snyder et al. 74BAAS6, 220.

338. Stasinska $73 A A 22,355$.

339. Stecher et al. 73MN161, 305.

340. Stecher et al. $74 M N 168,23 \mathrm{p}$.

341. Stecher et al. $74 M N 168,51 \mathrm{p}$.

342. Stephens et al. 73ApJ186, 165.

343. Strel'nitskij 74Uspekhi Fiz.Nauk 113, 463.

344. Strel'nitskij $73 A Z h 50,1133$.

345. Solomon et al. 75ApJ199, L79.

346. Solomon et al. 73ApJ185, L63.

347. Solomon et al. 73ApJ180, L89.

348. Spencer et al. 75ApJ199, L111.

349. Spitzer et al. 73ApJ186, L23.

350. Spitzer et al. 73BAAS, $5,4$.

351. Spitzer et al. 73ApJ181, L116.

352. Spitzer et al. 74ApJ191, L127.

353. Sullivan $73 A p J$ Sup25, 393.

354. Sume et al. 75AA39, 435.

355. Takano 74PASJ26, 189.

356. Takay anagi $73 P A S J 25,327$.

357. ter Haar et al. 74Rep. Progr. Phys.37, 481.

358. Thaddeus et al. 74ApJ192, L33.

359. Tiemann 74J.Phys.Chem.Ref.Data 3,259.

360. Troland et al. 74ApJ194, 43.

361. Tucker et al. 74BAAS6, 341.

362. Tucker et al. $73 A p J 186, \mathrm{~L} 13$.

363. Tucker et al. 74ApJ193, L115.

364. Turner 74JRASC68, 55.

365. Turner $74 A S S 29,247$.

366. Turner 74ApJ193, L83.
367. Turner $73 A p J 186,357$.

368. Turner et al. $75 A p J 198,71$.

369. Turner et al. 74ApJ194, 525.

370. Turner et al. 73BAAS5, 31.

371. Turner et al. 74ApJ187, L59.

372. Turner et al. 73BAAS5, 420.

373. Turner et al. 75ApJ198, L125.

374. Turner et al. 74BAAS6, 443.

375. Turner et al. 73ApJ186, 123.

376. Valtz et al. 73AL13, 23.

377. Varshalovich et al. 75AZh52, 6.

378. Waak et al. 74ApJ189,67.

379. Wahlgren et al. 73NatPS246, 4.

380. Wallerstein $73 A L 15,83$.

381. Watson 73ApJ183, L17.

382. Watson $73 A p J 182, \mathrm{~L} 73$.

383. Watson 73ApJ181, L129.

384. Watson 74ApJ189, 221.

385. Watson 74ApJ188, 35 .

386. White 73BAAS5, 421.

387. White et al. $75 M N 170,37 \mathrm{p}$.

388. Whiteoak 74PASA2, 248.

389. Whiteoak et al. 75ApJ195, L81.

390. Whiteoak et al. $75 M N 172,9 \mathrm{p}$.

391. Whiteoak et al. 74MN169, 59p.

392. Whiteoak et al. 74AA36, 245.

393. Williams $74 O b s 96,66$.

394. Wilson 74AA31, 83.

395. Wilson $73 A A 25,329$.

396. Wilson 72CIAL17, 447.

397. Wilson et al. 74PASP86, 602.

398. Wilson et al. 73AA22, 473.

399. Wilson et al. 73BAAS5, 22.

400. Wilson et al. 74ApJ191, 357.

401. Wilson et al. 73ApJ179, L107.

402. Wink et al. 73AA22, 251.

403. Winnberg et al. $73 A L 13,125$.

404. Winnberd et al. 73NatPS243, 78.

405. Winnewisser et al. 75ApJ200, L33.

406. Wynn-Williams et al. $74 A p J 187,41$.

407. Yngvesson et al. 73BAAS5, 476.

408. Yngvesson et al. 73BAAS5, 477.

409. Yngvesson et al. 75ApJ195, 91.

410. Zuckerman et al. 74ApJ189, 217.

411. Zuckerman et al. 74ApJ192, L149.

412. Zuckerman et al. 73BAAS5, 420.

413. Zuckerman et al. 75ApJ199, L35.

414. Zuckerman et al. 74ARAA12, 279.

415. Zuckerman et al. 75ApJ197, 571.

416. Zuckerman et al. 75ApJ197, 123.

417. Zuckerman et al. 74BAAS6, 443.

418. Zuckerman et al. 75ApJ196, L99.

\section{EXTRAGALACTIC RADIO ASTRONOMY}

(Y.N. Parijskij and S. Pustilnik)

For the last years no major changes have occurred in the development of extragalactic radio astronomy. The main flow of information has been supplied from the high resolution mapping 
of sources of different nature - compact and extended (Cambridge; Westerbork; NRAO, U.S.). These maps have given rise to new concepts on the energy transportation from the nucleus region to the extended components of radio galaxies. A new class of 'tailed' radio galaxies has been discovered, and extremely large objects have been found. There is some progress in the mapping of very compact objects with VLBI techniques, but the general picture of the early stages of evolution of radio events in QSR and RG is not yet clear.

Many new observations of variable radio sources available now, including $408 \mathrm{MHz}$ positive results and short-time scale phenomena up to a fraction of an hour, have been obtained. Permanent interest is taken in BL Lac-type objects. The general trend in this field is a rapidly increasing cooperation between different observatories and between different domains of the e.m. spectrum.

Much progress in the accuracy of position measurement is evident; the radio position now can be certainly more accurate than the optical one. New attempts are being made now to achieve an accuracy adequate to optical identifications of a great number of radio sources. Some new accurate observations of the anisotropy of the Black Body radiation are available, but they are not at the level of the extreme predictions of the Big Bang cosmology as yet. The number of the catalogued sources increases rapidly, approaching that in optical catalogues. Some very deep surveys have been made and new short wavelength surveys are in progress. References to all the subjects mentioned above can be found below.

\section{REFERENCES}

Small remarks available in some sections; frequency is given in $\mathrm{GHz}$ and flux density in $\mathrm{Jy}$.

1. Surveys Freq. min. flux

1.1 Shimmins et al. 72AJP Sup

23.

1.2 Maslowski 72Act Astr. 22, 227.

5.0

1.3 Pauliny-Toth et al. 72AJ77, 797.

1.4 Gorshkov et al. 72Izv. Krymsk. Astrofiz.Obs45, 176.

1.5 Shimmins et al. 72 AJP Sup 26.

1.6 Colla et al. 73AA Sup11, 291.

1.7 Davies et al. 73AJPSup28. .41, 0.1

1.8 Bolton et al. 73AJPSup30. 27, 0.22

1.9 Fanti et al. 74AASup18, 147.

1.10 Katgert et al. 74AA35,

393.
1.11 Sutton et al. 74AJPSup

$.41,0.5$ 33.

1.12 Rinsland et al. 74AJ79, 1129.

1.13 Katgert $75 A A 38,87$.

1.14 Bolton et al. 75AJPSup 34.

1.15 Bolton et al. 75AJPSup 34. 1.40 .09 5.0 8.6

$2.7,0.15$

$.61,1.4, .007$

$.41,0.2$

$1.4,0.18$

$1.4,0.006$

$2.7,0.5$

$2.7,0.22$

1.16 Wall et al. 75AJPSup34. 2.7, 0.5

1.17 Shimmins et al. 75AJPSup 34. $2.7,0.5$

1.18 Pearson $75 M N 171,475$. $0.14,1.4,0.009$

\section{Flux Density Measurements}

(i) Optically selected sources: Detections and measurements

Object; Freqs.

2.1 Tovmassian 72AJ77, 705. Markarian Gal. 30

2.2 Katgert et al. $73 A A 23$, 171.

2.3 Allen et al. $73 A A 25$, 187.

QSO's 1.4

Gal 1.4

2.4 McCutcheon 73AJ78, 18. Gal 6.6

2.5 Berkhuijsen et al. 73AL13, 169.

M31 2.7

2.6 Slee 72PASA2, 159 . Gal

2.7 Ekers et al. 73AA24, 247. E Gal 5.0

2.8 Wright et al. $72 C I A L 17$, Interacting Gal 6.5, 585.

2.9 Sulentic et al. $73 A p J 182$, L17.

2.10 Thomasson et al. $73 M N$ 162, 295.

2.11 Peckham 73NatPS246, 54. 14

2.12 Gnibert 73AA29, 335.

2.13 Tovmassian et al. $73 \mathrm{AL}$ $15,97$.

Chains, Groups

Mark. Gal .41

Cyg A

M31 companions 1.4

Normal 0.43

Spirals

2.14 Tovmassian 73Astrofiz 9 , 2.

2.15 Le Sqrieren et al. $74 A A$ 31, 447.

Clusters of galaxies

Gal 4.8

2.16 Wright $74 M N 167,273$. Gal $2.7,5.0$ 
2.17 Tomasson et al. 73 Radiofizika 16, 1342.

2.18 Israel et al. 74AA32, 363.

2.19 Webber 74PASP86, 223.

2.20 Owen $74 A J 79,427$.

2.21 Kellermann 74ApJ194, L135.

2.22 Efanov et al. 74Soviet Astron.18, 4.

2.23 Kaftan-Kassim et al.74AA $33,343$.

Mark. Gal .41 M33 H Il-regions 1.4

Abell 1314

Abell Clusters

1.4

Cen A

$3 \mathrm{C} 27378$

Stephan Quintet $0.32,0.43,0.61$

2.24. Kaufman et al. $74 M N$ $169,15 \mathrm{P}$

2.25 Hitchmeier et al. $74 \mathrm{AL}$ 15, 209.

NGC5128 22

$3 \mathrm{Zw} 216,2.7,5$, $8.9,9.9$

2.26 Tovmassian et al. 74PASP Mrk galaxies 86, 649. .43

2.27 Guthrie $74 M N 168,15$. Brightest galaxies in Clusters 1.4

2.28 Gerhart 74 Nat 249, 743 . OH471 80Q172 $0.017-85$

2.29 Colla et al. 75AASup20, 1.

Gal $0.41,1.4$, 5.0

2.30 Kaufmann et al. 75ASS Sersic galaxies 32, L25.

2.31 Heeschen et al. 75ApJ $196,347$. 22

E-galaxies 31 , 86

2.32 Owen $75 A J 80,263$.

Abell Clusters 2.7

(ii) Other flux density measurements

Freq.

2.33 Lipovka 72Soviet Astron. 16, 5.

2.34 Conklin et al. $72 \mathrm{ApJ177}$, 303. 7.7

2.35 Zabolotny et al. 72Soviet Astron. 16, 5.

2.36 Webber 72AJ77, 625. $0.75,1.4,2.7$,

2.37 Wall 72AJPSup24, 1 . 47, 64, 1.4, 50

2.38 Schillizzi et al. 72AJP25, 545.

$08,41,5.0$

2.39 Tovmassian et al. 72 Astron Tsirk. 715.

2.40 McGee et al. 72AJP25, 581.

2.41 McGee et al. 72AJP25, 613.

2.42 Stull et al. $73 A p J 13,73$. 8.0

2.43 Shimmnins et al. 73AJP 26, 93.

2.44 Slec et al. 73AJPSup27. 0.08

2.45 Stull $73 A J 78,285$. 0.43

2.46 Finlay et al. $73 A J P 26$, 389.

0.03
2.47 Welch et al. 73PASP85, 456.

2.48 Andrew et al. $73 \mathrm{ApJ185}$, 137. 61-11

2.49 Lipovka 73Astrofiz.Issled. Izv SAO5, $30 . \quad 7.7$

2.50 Kellermann et al. $73 A J 78$, 828.

11

2.51 Roger et al. 73AJ78, 1030 . $\quad 0.01,0.022$

2.52 Bogod et al. 73Radiofizika 16, 765.

2.53 Crovisier et al. 74AA34, 285.

2.54 Pauliny-Toth et al. 74AA 35,421 .

2.55 Soboleva 74Astron.Tsirk $826,1$.

2.56 Bridle et al. $74 A J 79$, 1000.

2.57 Condon et al. 74AJ79, 1220.

2.58 Dent et al. 74AJ79, 1232.

259 Maslowski 74AA36, 395, 15

2.60 Rather 74PASP86, 601. 210

2.61 Grueff et al. 75AASup19, 117. 50

2.62 Efanov et al. 74Astron. Tsirk 842, 1.

2.63 Fanti et al 75AASupl9 $143 . \quad 5.0$ 22

2.64 Cogdell et al. $75 A p J 196$, 363.

2.65 Colla $75 A A 38,209$. 1.4, 5.0

2.66 Bolton et al. 75AJPSup34,

2.67 Bolton et al. 75AJPSup 34. $2.7,5.0$ $2.7,5.0$

2.68 Wall et al. 75AJPSup34. 2.7, 5.0

2.69 Shimmins et al. 75AJPSup 34.

2.70 Sharp et al. 75AJ80, 335. 0.36

2.71 Clegg et al. 74Nat249, 530.

2.72 Zabolotnyj et al. 75Pisma AZh $1,14$.

$\begin{array}{lll}\text { ee } \text { et al. } 75 \text { AJPSup36. } & 0.08\end{array}$

2.74 Rowan-Robinson et al. $75 M N 172,603 . \quad 190$

2.75 Efanov et al. 75Izv. Kryms. Astrofiz Obs.53, $144 . \quad 77$

(iii) Flux density scales and absolute measurements

2.76 Dent 72ApJ177, 93.

2.77 Wills $73 A p J 180,335$.

2.78 Janssen et al. 74AA33, 373.

2.79 Ross et al. 75MN170, 115. 
2.80 Viner $75 A J 80,83$.

2.81 Sramek et al. 75ApJ196, $339 . \quad$ Mark.Gal 5.0

2.82 Jaffe et al. 75AASup21, Abell Clusters $137 . \quad 1.4$

\section{Position Measurements}

\section{(i) Better than $1^{\prime \prime}$}

3.1 Douglas et al. 73AJ78, 1. Texas

3.2 Crowther 73Nat 243, 25.

3.3 Brosche et al. 73ApJ183, 805.

NRAO

3.4 Ghigo et al. 73AJ78, 848. Texas

3.5 Rogers et al. 73ApJ186, 801.

3.6 Adgie $74 A J 79,846$.

3.7 Grueff et al. 75AASup19, 117.

3.8 Fanti et al. 75AASup19, 143.

RRE B2

3.9 Warner et al. 75PASP87, 103.

(ii) Better than 5"

3.10 Hoskins et al. 72AJP25, 559.

Molonglo

3.11 Sharp $75 A J 80,335$.

3.12 Haynes et al. 75AJ80, 673.

Texas

NRAO

(iii) Position comparisons

Note

3.13 Argue et al. $73 A L 14,99$. opt/radio

3.14 Véron et al. $75 A A 42,1$. opt/radio

\section{Brightness Distribution Measurements}

(i) Pencil Beam

4.1 Durdin et al. 72AJ77, 637. M31

4.2 Broten 72AJP25, 599. Large Magellanic

4.3 Wright $74 M N 167251$. Interacting Galaxies

4.4 Kaftan-Kassim et al. 74AA Stephan Quintet 33, 343.

4.5 Berkhuijsen et al. 74AA 34, 173. Region

4.6 Baker et al. 74Nat252, 552. M31

4.7 Owen 75AJ80, 263.

4.8 Dennison et al. 75PASP87, 83.

4.9 Kap-herr et al. 75AA41, 115.

DA240

Abell Clusters

M31, M33

M81 (ii) Interferometric: Baselines $<3 \mathrm{~km}$

4.10 Van der Kruit et al. $72 A A 21,169$.

4.11 Yip et al. $72 A p J 177$, $291 . \quad$ Cyg A

4.12 Riley $73 M N 161,167$. 3C

4.13 Baldwin et al. $73 M N 161$, $127 . \quad$ NGC

4.14 Becker et al. 73NatPS244,2048 + 31 138.

4.15 Harris $73 M N 163,19$. 3C52, 3C192.

4.16 Miley 73AA26, 413 . 3C

4.17 Wellington et al. 73Nat 244, 502.

NGC1265

4.18 Arp $73 A p J 183,791 . \quad$ Spiral galaxies

4.19 Miley et al. $73 A A 28,359.3 \mathrm{C}$

4.20 Burke et al. 73AA28, Interacting 379.

4.21 Van der Kruit 73AA29, Bright 231.

4.22 Van der Kruit 73AA29, Bright 249.

4.23 Donivom et al. $74 \mathrm{ApJ}$ $187,11$.

4.24 Slingo $74 M N 168,307$. 3C

4.25 Högbom et al. 74AA34, 341 .

4.26 Schwarz et al. 74AJP27, 563.

PKS

4.27 de Bruyn et al. 74AA33, Seyfert 351.

4.28 Sramek et al. 74ApJ191, 633.

galaxies nuclei

4.29 Wilkinson 74 Nat 252 ,

661. M87 jet

4.30 Kaufmann et al. $74 M N$ 169, 15.

4.31 Riley $75 M N 170,53$.

4.32 Colla et al. 75AASup20, 1.

4.33 Fanti et al. 75AASup19, 143.

spirals

Clusters of galaxies C

Mrk 6

Cen A

$4 \mathrm{C}$

$\mathrm{Gal}$

4.34 Kaftan-Kassim et al. 75 Stephan Nat 253, 176.

4.35 Colla et al. $75 A A 38,209$. $\mathrm{B} 2$

4.36 Schillzzi et al. 75AA40, 221.

4.37 Jaffe et al. 75AASup21, 137.

NGC7385

4.38 Haynes et al. 75AJ80, Normal 673.

4.39 Stull et al. 75AJ80, 559. 3C

4.40 Israel et al. 75AA40, 421.
4.41 Schilizzi 75MRAS79, 1 .

(iii) Interferometric: Baselines $>3 \mathrm{~km}$

4.42 Clark et al. 72AZh16, 4.

4.43 Erickson et al. 72ApJ177, 101. 
4.44 Peckman et al. 72NatPS240, 76.

4.45 Critchley et al. 72MN160, 271.

4.46 Wraith $72 M N 160,283$.

4.47 Wilkinson $72 M N 160,305$.

4.48 Purton et al. 72MN159, 15.

4.49 Browne et al. 73Nat 244, 19.

4.50 Clark et al. 73ApJ182, L57.

4.51 Legg et al. 73Nat 244, 18.

4.52 Riley et al. 73MN164, 271.

4.53 Matveenko et al. 73Soviet Astron.17, 6.

4.54 Shapiro et al. 73ApJ183, L47.

4.55 Kellermann et al. 73ApJ183, L51.

4.56 Northover $73 M N 165,369$.

4.57 Willis et al. 74Nat250, 625 .

4.58 Wilkinson et al. $74 M N 168,515$.

4.59 Rogers et al. 74ApJ193, 293.

4.60 Pooley et al. 74MN169, 477.

4.61 Bash et al. 74AJ79, 1341.

4.62 Hargrave $74 M N 168,491$.

4.63 Turland $75 M N 170,281$.

4.64 Wittels et al. 75ApJ196, 13.

4.65 Turland $75 M N 172,181$.

4.66 Cotton et al. 75AJ80, 353.

4.67 Rellarmann et al. 75ApJ197, L113.

4.68 Niell et al. 75ApJ197, L109.

4.69 Fomalont et al. 75Nat 257, 99.

4.70 Spinrad et al. 75ApJ198, 7 .

\section{(iv) Occultation}

4.71 Abramyan 72Soviet Astron.16, 4.

4.72 Kapahi et al. 73AL14, 31 .

4.73 Kapahi et al. 73AJ78, 673, 805.

4.74 Joshi et al. $73 A J 78,1023,1141$.

(v) Scintillation

4.75 Shishov 72Soviet Astron. 16, 6 .

4.76 Harris $73 A J 78,369$.

4.77 Shishov 72IVUZR15, 1277.

4.78 Vitkevich et al. 72 Trudy Fiz.Inst.Akad. Nauk SSSR62, 42.

4.79 Hewish et al. 74Nat 252, 657.

4.80 Harris $74 A J 79,1211$.

4.81 Cohen et al. 74ApJ192, 193.

4.82 Condon et al. $75 A p J 197,31$.

\section{Polarization Measurements}

(i) Integral linear polarization

Freq.

5.0 Berge et al. $72 A J 77,810.1 .4,1.6,5.0$

5.1 Gauss et al. 73ApJ179, 439.

5.2 Efimov et al. 72Izv. Krymsk Agtrofiz.Obs. 46, 3.

$1.25-1.45$

5.3 Strom $73 A A 25,303$.

5.4 Bignell et al. 73AJ78, 536.
5.5 Bignell 73AJ78, 557.

$6.7,11$

5.6 Wardle et al. 74ApJ189, 399.

5.7 Conway et al. $74 M N 168$, 137.

$2.7,8.1$

41-14

5.8 Haves et al. $74 M N 169$, 117.

5.9 Vallée et al. 74ApJ193, 303.

97

66,14

5.10 Wardle et al. $74 A \mathrm{pJ} 194$, 249.

5.11 Scielstad et al. 75AJ80, 271.

5

5.12 Ekers et al. $75 A A 38,67.1 .4$

5.13 Gardner et al. 75AJP35. 1.4, 1.7, 2,7, 5.0

(ii) Linear polarization distribution

5.14 Fomalont $72 A L 12,187$.

5.15 Yip et al. $72 A p J 177,291$.

5.16 Kronberd et al. $73 A L 14,25$.

5.17 Mirovsky et al. 73AZh17, 5.

5.18 Miley $73 A A 26,413$.

5.19 Miley et al. $73 A A 28,359$.

5.20 Högbom et al. 74AA34, 341 .

5.21 Soboleva 74Soviet Astron 18, 6.

5.22 Baker et al. 74Nat 252, 552 .

5.23 Schwarz et al. 74AJP27, 563.

5.24 Stull et al. 75AJ80, 559 .

(iii) Circular polarization

5.25 Seaquist 73AA22, 299.

5.26 Seaquist et al. 73NatPS242, 20.

5.27 Seaquist et al. $74 A J 79,918$.

5.28 Ekers 75AA38, 67.

5.29 Roberts et al. 75AJP28, 325.

6. Variable Source Observations

Freq.

6.1 Romano 72Mem.Soc.Astron. Italiana.Nuova Ser.43, 309. Opt

6.2 Gorshkov et al. 72 Soviet Astron. 16, 4. $6.7,8.0,11$

6.3 Tsesevich 72 Astron. Tsirk. $688,7$.

6.4 Babadzhanjanz et al. 72 Astron. Tsirk 701, 1.

Opt

6.5 Medd et al. 72MRAS77, 109.

Opt

6.6 Hunstead $72 A L 12,193$.

$6.7,11$

6.7 Haden-thorn 72Astron.

Tsirk.714, 5.

.41

Epstein et al. $72 \mathrm{ApJ178,}$

L51.

Opt

6.9 Dent et al. $72 A 577,819$. 7.8

6.10 Lu 72AJ77, 829. Opt

6.11 Efanov et al. 72Izv.Krymsk. Astrofiz.Obs. 45, $172 . \quad 36$ 
6.12 Rieke et al. $72 \mathrm{ApJ177}$, L115.

IR

6.13 Visvanathan $73 A p J 179$, 1.

6.14 Craine et al. $73 \mathrm{ApJ} 179$, L53.

6.15 Visvanathan et al. $73 \mathrm{ApJ} 179,721$.

6.16 Weistrop 73NatPS241, 157, 243, 144.

6.17 Godsmith et al. $73 \mathrm{ApJ}$ 180,661 .

6.18 De Yound et al. 73ApJ 180, L61.

Opt

Opt

Opt

Opt

Opt

$2.7,8.1$

6.19 Dent et al. 73AJ78, 163. 31

6.20 Pfau 73Inform. Bull. Variable Stars 787.

Opt

6.21 Angione 73AJ78, 353. Opt

6.22 Babadzhanyants et al. 73 Trudy Astron. Obs.

Leningrad 29, 72.

6.23 Kellermann et al. $73 \mathrm{ApJ}$

6.24 Epstein 73IAU Circ. 2519.

Opt

6.25 Bignell 73AJ78, 557. 6.6,11

6.26 Bignell $73 A J 78,557 . \quad 6.6,11$

6.27 Eggen $73 A p J 186, \mathrm{Ll}$. Opt

6.28 Shapiro et al. $73 \mathrm{ApJ} 183$, L47.

6.29 Kellermann et al. $73 \mathrm{ApJ}$ 183, L51.

6.30 Babadzhanyants et al. 74 Trudy Astron. Obs. Leningrad 30, 69.

Opt

6.31 Crovisier et al. 74AA30, Opt B20912
175.

6.32 Tabara et al. 74Tokyo Astron. Bull. 2nd Ser.228, 2633.

6.33 Riplinger $74 A p J 191$, L109. Opt

6.34 Haves et al. $74 M N 169$, 117.

6.35 Seielstad $74 A p J 193$, 55.

6.36 Bramdie et al. 74Nat252, 212.

6.37 Usher et al. 74Nat 252, Opt PKSOO48 365. $-097$

6.38 Dent et al. 74AJ79, 1232 .

6.39 Miller et al. $74 A J 79, \quad$ Opt PKS1514 1352. $-24$

6.40 Malumyan et al. 74 Astrofiz 10, 4.

\section{$.413 \mathrm{C} 454.3$}

6.41 Sramek et al. 74ApJ101, L13.

6.42 Fogarty et al. 74ApJ191, 305.

$2.7,5,8$ Mrk 348

90 NGC 1068

6.43 Hitchmeier et al.74ApJ 15, 209.
$5,8,9,9.9$

$3 \mathrm{Zw2}$
6.44 Andrew et al. 74ApJ191, Opt Radio 51.

Bllac

6.45 Frohlich et al. $74 M N 168$, 417.

Opt OJ 287

6.46 Milone 74PASP86, 899. Opt Bllac

6.47 Selmes et al. $75 M N 170$, 15.

Opt

6.48 Brandie et al. 74Nat252, 212.

6.49 Adgie et al. $75 M N 170$, 31. $1,2.7,8.0$

6.50 Markava et al. 74Astron. Tsirk843, 1. Opt

6.51 Kurochkin 74Astron. Tsirk. $844,1$.

Opt

6.52 Seielstad et al. 75AJ80, 271. 5.0

6.53 Sanamyan et al. 75 Astrofiz 11,1 .

6.54 Sanamyan et al. $\quad 0.41$ 75 Astrofiz 11, 1 .

6.55 Markova 75Astron. Tsirk. $862,1$.

Opt

6.56 Markova 75Astron. Tsirk. 856, $4 . \quad$ Opt

6.57 Onrassine $75 \mathrm{Nat} 254,125$. Opt

6.58 Saakyan 75Astron. Tsirk. $861,7$.

6.59 Fogarty et al. 75Nat 254, 124. Opt

6.60 Ekers et al. $75 A A 38,67.1 .4$

6.61 Price et al. 75Nat 255,467.10.7

6.62 Craine et al. 75PASP87, 123.

6.63 Smith et al. 75PASP87, 149.

Opt

Riplinger $75 A J 80,669 . \quad$ Opt

6.65 Veron et al. 75AA39, 281.

6.66 Veron $75 A A 41,423$. Opt

6.67 Dowison 75ApJ196, L133. Opt

6.68 Litter et al. 75ApJ199, L133. Opt

6.69 Winkler et al. 75ApJ199, L139. X-ray

6.70 Stamard et al. 75 Nat 255, 384.

\section{Microwave Background Measurements}

7.1 Parijskij 72Soviet Astron. $16,6$.

7.2 Hegyi et al. 73BAAS5, 34.

7.3 Boynton et al. 73ApJ181, 243.

7.4 Carpenter et al. 73ApJ182, L61.

7.5 Muehlner et al. 73Phys.Rev.D7, 326.

7.6 Bahcall et al. 73ApJ182, L95.

7.7 Parijskij 73ApJ180, L47.

7.8 Parijskij 73Soviet Astron.17, 3.

7.9 Antonov 73IVUZR16, 759. 
7.10 Stankevich 74Soviet Astron. 18, 1.

7.11 Boynton et al. 74Nat 247, 528.

7.12 Gush 74Can.J.Phys. 52, 554.

7.13 Robson et al. 74Nat251, 591.

7.14 Blair 74IAU Symp.63, 143.

7.15 Partridge 74IAUSymp.63, 143.

7.16 Boynton 74IAUSymp.63, 163.

7.17 Rozental 74Novye probl.astrofiz. Trudi Mosk. Univ. 91, 11.

7.18 Hegyi et al. 74ApJ190, 543.

7.19 Mather et al. 74IEEE Microwave Theory Techn. 22, 12, Part 1, 1046.

7.20 Wall et al. 75MN171, 9.

7.21 Woody et al. 75PRL34, 1036.

\section{Miscellaneous Observations}

8.1 Rieke 72ApJ176, L61.

IR of BL Lac-

8.2 Carswell et al. 73Nat242, 394.

type

8.3 Wampler et al. 73Nat 243, 336.

OH 471

8.4 Wrich $73 A L 13,89$.

00172

8.5 Tritton et al. $73 M N 162$, 31.

B2 $1101+38$

$4 \mathrm{C} 31.63$

8.6 Becklin et al. $73 \mathrm{ApJ} 181$, L27.

NGC253

8.7 Sargent 73ApJ182, L13. Redshifts of $4 \mathrm{C}$ sources

8.8 Babadzhanjants et al. 72 Astrofiz8, 4.

SyG variability

8.9 Baldwin et al. 73ApJ185, 739.

QSO candidates

8.10 Wampler et al. 73Nat246, 203.

Double QSO

8.11 Riruchi et al. 73PASJ25, OJ287 optic 555.

8 radio

8.12 Pronik 74Soviet Astron. 18, 3 . NGC1275

8.13 Argue et al. $74 M N 168,1$. 4C 11.50

8.14 Schmidt $74 A p J 193,505$. Redshifts of $4 \mathrm{C}$ QSSs

8.15 Schmidt $74 A p J 193$, 509. Spectroscopy of faint blue objects

8.16 Baldwin et al. 74ApJ193, Optical lines of 513. OQ172

8.17 Stockton 74Nat 250, 308. Galaxy near 4C

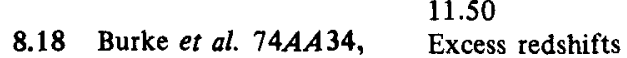
445.

8.19 Penston et al. 74MN169, Seyfert $357 . \quad$ galaxies

8.20 Teerikorpi 74Nat 252, Redchifts of 110. Markarian galaxies

8.21 Kinman et al. 74ApJ194, Optical 8 IR L1. M87 jet
8.22 Pronik 74Soviet Astron. Nucleus of 18, $6 . \quad$ NGC 1275

8.23 Andrews et al. 74MN168, Multicolour 7. photometry APLib PKS 0521-36

8.24 Disney et al. 74ApJ194, Redshift nature L79.

of AP Lib

8.25 Strittmatter $74 A p J 190$, Spectroscopy of 509.

8.26 Oke 74ApJ189, L47. Spectrophotometry of OQ 172 SO11471

8.27 Schōnfelder et al. 74ApJ $\gamma$ - ray Back191, L1

8.28 Denisyuk et al. 74Astron. Tsirk. 837, 1. ground

Mark 380

8.29 Baldwin 75ApJ195, L55. Opt.spectrum BL Lac

8.30 Carsuell et al. 75ApJ195, Optical lines of 269.

OH 471.

8.31 Osterbrock et al. 75ApJ Broad lines in 197, L41.

8.32 Grindlau et al $75 \mathrm{ApJ}$ radio galaxies 197, L9. $\gamma$ - rays from Cen A

8.33 Baldwin 75ApJ196, L91. Optical lines in 3C 273 S 0736 $+01$

8.34 Grewing et al. 75AA42, Pair NGC3067/ 119.

$3 \mathrm{C} 23221 \mathrm{~cm}$ absorption

8.35 Osterbrock et al. 75ApJ Emission-Line $197,535$.

Spectrum of Cyg A

8.36 Grindlay et al. 75ApJ197, Broad Balmer L41. Lines in Radiogalaxies

8.37 Kinman 75ApJ197, L49. Nebulosity in BL Lac

8.38 Tung Chan 75ApJ198, Emission Line 45. strengths in QSOs

8.39 Grindley et al. $75 A p J 197, \gamma$-rays from L9. Cen A

8.40 Margon et al. 75ApJ197, X-ray spectroscopy 25.

Extragalactic radiosources

8.41 Boksenberg et al. $75 M N$ 172, 289. Spectroscopy of $3 \mathrm{C} 273$.

8.42 Morgan et al. 75MN171, Ultraviolet 599.

colours of quasars

\section{Optical Identifications}

9.1 Véron $72 A A 20,471$.

9.2 Merkelijn $72 A J P 25,451$.

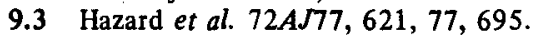


9.4 Argue et al. 72MN160, 197.

9.5 Tritton $72 M N 158,277$.

9.6 Spinrad et al. 73ApJ179, L71.

9.7 Glass $73 M N 162,35$.

9.8 Bergamini et al. 73AA23, 195.

9.9 Gent et al. 73Nat 241, 261.

9.10 Argue et al. 73NatPS241, 139.

9.11 Peterson et al. 73AL13,187.

9.12 Parkes et al. 73MN162, 117.

9.13 Agnew et al. 73PASP85, 162.

9.14 Fanti et al. 73AA24, 69.

9.15 Browne et al. 73Nat 244, 146.

9.16 Pauliny-Toth et al. 73AA27, 475.

9.17 Véron et al. 73AL14, 237.

9.18 Welch et al. 73PASP85, 456.

9.19 Wills et al. 73AJ78, 521.

9.20 Véron et al. 73AA28, 319.

9.21 Wampler et al. 73Nat 246, 203.

9.22 Carswell et al. 73Nat 246, 89.

9.23 Tritton et al. 73MN165, 245.

9.24 Tritton et al. 73MN165, 253.

9.25 Baldwin et al. 73MN165, 259.

9.26 Bolton et al. 73AJPSup30.

9.27 Wall $73 A L 15,101$.

9.28 Joshi et al. $73 A J 78,1023,1141$.

9.29 Kristian et al. 74ApJ191, 43.

9.30 Peterson et al. 73AL15, 109.

9.31 Faranoff et al. $73 A L 15,117$.

9.32 Brandie et al. 74AJ79, 903.

9.33 Katgert et al. $74 A A 35,393$.

9.34 Grueff et al. $74 A A 35,491$.

9.35 Johnson 74AJ79, 1006, 1123.

9.36 Wills et al. 74ApJ190, L97.

9.37 Longair et al. 75MN170, 121 .

9.38 Adgie et al. $75 M N 170,31$.

9.39 Colla et al. 75AASup20, 1.

9.40 Richter $75 A N 296,65$.

9.41 Grueff et al. 75AASup20, 57.

9.42 Bystedt $75 A A 39,155$.

9.43 Colla 75AA38, 209.

9.44 Bolton et ql, 75AJPSup34.

9.45 Shinmins et al. 75AJPSup34.

9.46 Warner et al. 75PASP87, 103.

9.47 Vidal 75AA42, 145.

9.48 Lelievre et al. 75AA42, 293.

9.49 Notni et al. 75AN296, 197.

9.50 Spinrad et al. 75ApJ198, 7.

9.51 Schilizzi 75MRAS79, 75.

\section{Interpretation of Spectral Data}

10.1 Gnedin et al. 72Pisma Zh.Exp. Teor. Fiz. 16, 45.

10.2 Wall 72AJPSup24, 49.

Non-exponential radio spectra Statistical analysis

10.3 McGee et al. 72AJP25, 619.

Sources of Large Magellanic Cloud

10.4 Kurilchik 73Soviet Astron. Haloes of double 17,1 .
10.5 Faranoff et al. $73 M N 161$, 393.

10.6 Braude et al. $73 A S S 20$, 59.

10.7 Grueff et al. 72Lab. Radioastron. CNR, Ist. Fis. Bologna, Separate Print.

10.8 Slingo $74 M N 168,307$.

10.9 Jones et al. $74 \mathrm{Nat} 250$, 472.

10.10 Brandie et al. $74 A J 79$, 903.

10.11 Braude 74ASS31, 203.

10.12 Condon et al. $74 A L 15$, 203.

Evolution of sources

Non-linear low-

frequency

spectra

Correl.spectral

index-optical

power

Radio sources

with very steep

spectra

Structure of

OH4718 OQ172

'Two-color' radio

analogue

Effects of dis-

tribution function

of relativistic

particles

Non-uniform

compact sources

\section{Interpretation of Variability Data/Compact Sources}

11.1 Dorschner et al. 72AN294, 65.

Model

11.2 Budden et al. 72Proc. Roy. Scintillation of Soc.London, SerA.330, extended Sources 65.

11.3 Alvarez et al. 73ApJ179, 391.

3C 279

11.4 Nicolson 73NatPS241, 90.

Variable sources, non-variable sources.

11.5 Chertoprud et al. 73NatPS 3C273 Cyclicity 242, 70 .

11.6 Chertoprud et al. 73ApJ 182, L53.

11.7 Jones et al. 73ApJ186, 791.

3C273 Independent Pulses Decimetric flux variations

11.8 Ginzburg 73Nat 246, 415.

11.9 Babadzanyants et al. 74 Astron. Tsirk. 817, 3.

Intergalactic gas and flares Periodicity in $3 \mathrm{C}$ 371.

11.10 Jones $74 A p J 191, \mathrm{~L} 15$.

11.11 Elliot et al. 74ApJ192, L3.

11.12 Cocke et al. 75ApJ195, 279.

Angular radius of

PKS $0735+178$

Accreting black

hole model

Low-frequency

variability

\section{Interpretation of Structure Data}

12.1 Allen et al. 73Nat 241, 260. Interacting

galaxies

12.2 Guthrie 73ASS23, 391. Extragalaetic

$\mathrm{X}$-ray sources

12.3 Van der Kruit $73 A A 29$, 263.

Bright spiral galaxies

12.4 Vorontson-Velyaminov 74 Comet-like Astron. Tsirk. 816, $1 . \quad$ galaxies 
12.5 Browne et al. 74Nat 252, High redshift 209.

\section{QSOs}

12.6 Turland 75MN170, 281. M87

12.7 Aizu 75Progr. Theor.Phys. Extended sources 53, 893.

13. Interpretation of Polarization Data

13.1 Mitton $73 A p J 13,19$.

13.2 Melrose 72PASA2, 140.

13.3 Fukui 73PASJ25, 181.

13.4 Sazonov $72 A S S 19,3,25$.

13.5 Pacholczyk 73MN163, $29 \mathrm{P}$.

13.6 Nelson 73PASJ25, 489.

13.7 Bignell 73AJ78, 1058.

13.8 Conway et al. $74 M N 168$, 137.

13.9 Tokarev 74Radiofizika16, 1789.

13.10 Pacholczyk et al. 74ApJ 192, 591.

13.11 Pacholczyk 75ApJ196, 125.

13.12 Kolobov et al. 75Soviet Astron. 19, 169.

13.13 Stocke et al. 75MN172, 567.

Internal Faraday rotation

Self-absorbed synchrotron

sources

Internal Faraday

rotation

Circular polari-

zation synchro-

tron source

Circular repola-

rization

Rotation measureredshift

Rotation measurecircular polarization

Polarization vs wavelength

Faraday depolarization

Absorption effects on circular repolarization

Oscillatory behaviour of intensity

Degree of homogeneity of magnetic field

Synchrotron emission in plasma

14. Redshift Dependent Properties of Sources

14.1 Richter $73 A p J 13,63 . \quad$ Ang. size

14.2 Bahcall et al. 73ApJ179, Hubble diagram 699.

14.3 Rowan-Robinson $73 A A$ 23, 331 .

$14.4 \cdot \operatorname{Ross} 73 A A 24,471$.

14.5 Zotov et al. $73 M N 162$, 127.

\section{QSO}

Two classes of quasars Redshift/Variation particle rest mass

QSO Luminosity evolution

14.6 Chiu et al. $73 A \mathrm{pJ} 181,295$. Two types of quasars

14.7 Dehren et al. 73AL14, 91. Quasar peculiar velocities

14.8 Jackson $73 M N 162,11$.

14.9 Nelson 73PASJ25, 489. Redshift-angular size Redshift-rotation measure 14.10 Evans 74ASS30, 299. Distance of BL Lac
14.11 Oke et al. $74 A p J 189$, L5.

14.12 Baldwin et al. $75 \mathrm{ApJ}$ 195, L55.

14.13 Thuan et al. 75ApJ201, 45.

15. Miscellaneous Correlations

15.1 Thiel $72 A S S 17,39$.

15.2 Lyutyi 72 Soviet Astron. 16,5 .

15.3 Ozernoy 72Soviet Astron. $16,6$.

15.4 Oemler et al. 72ApJ176, L47.

15.5 Kristian 73ApJ179, L61.

15.6 Evans 73NatPS241, 5.

15.7 Andrew et al. 73 NatPS 241, 158.

Distance of

BL Lac

Distance of BL Lac

Distance of BL Lac

15.8 Strom 73NatPS244, 2.

15.9 Burbidge et al. $73 A p J 182$, L47.

Double Source Orientation Optical/Radio Variability

Pairs Galaxy QSO

$\mathrm{QSO}$ in cluster of Calaxies QSO nuclei of Galaxies Ionization potential redshift

Chain of radio sources Depolarization/ Size

15.10 Grueff et al. 73AASup11, Spectral Index$41 . \quad$ Ratio of Radio to Optical Power

15.11 Browne et al. 73MN162, QSO' s/bright $21 \mathrm{P}$.

galaxies

15.12 Miller et al. $73 A p J 179, \quad \mathrm{BSO} /$ galaxies L83.

15.13 Hall et al. 73NatPS241, Optical Amplitu31.

des-Radio Spectra

15.14 Bogart et al. 73ApJ181, Clustering among 609.

clusters of galaxies and quasars

15.15 Morris et al. 73PASJ25, Depolarization295.

Luminosity

15.16 Bahcall et al. 73ApJ183, QSO-clusters of 777.

galaxies

15.17 Condon et al. 73ApJ183, 5C spectral L33.

15.18 Ames et al. 73ApJ14, Variability199.

Variability
luminosity

15.19 Basu 73Obs93, $184 . \quad$ Opt.variability radio spect.index

15.20 Rozyczka 73Act Astr.23, QSO-galaxies 233. coincidence

15.21 Hazard et al. 73Nat 246, Discrepant red205. shifts of QSO

15.22 Baldwin et al. 73MN165, X-ray clusters259. steep spectra

15.23 Guthrie 74ASS27, 489. $\mathrm{X}$-ray and radio emission by clusters of galaxies. 


\subsection{Owen $74 A p J 189$, L55 \\ 15.25 Tovmassian et al. 74 Astrofiz 10, 1.}

15.26 Bell 74ApJ194, 245.

15.27 Noonan 74Apji191, 301. Frequency of peakpower to peak

15.28 Tovmassian et al. $74 \mathrm{Nat}$ $250,474$.

15.29 Vallee $74 A J 79,1015$.

15.30 Ricke et al. 74ApJ192, L115.

Radio galaxies

in clusters

Polarization at

$6 \mathrm{~cm}$ and other parameters

Optical IRbeha-

vior OJ 287

Similar objects

15.31 Disney 74ApJ193, L103. BL Lac object in cluster of galaxies

15.32 Evans $74 O b s 94,50$.

15.33 Mchardy 74MN169, 527.

Radio magnitude redshift relation Clusters of galaxies; radio galaxies

15.34 Baldwin 74Obs94, 264. Radiosources in clusters

15.35 Riley $75 M N 170,53$.

15.36 Roeder 75Nat 255, 124.

15.37 Readhead et al. $75 M N$ 170, 393.

Brightest galaxy of cluster-radiosource

Angular diameter -redshift

Scintillating radio sourcesevolution

15.38 Battistini et al. 75AA40, Quasar-group of 217.

15.39 Swarup 75MN172, 501. galaxies

Anglular sizeflux density

15.40 Dolan 75ApJ16, 65.

15.41 Van den Bergh 75ApJ 198, L1.

Probability of associat. of pairs of different distances Quasar cluster association

16. Interpretation of Microwave Background Data

16.1 Layser et al. 73ApJ179, 361.

16.2 Noerdlinger 73PRL30, 761.

16.3 Dautcourt 74AN295, 123.

16.4 Anile 74ASS29, 415.

16.5 Rowan-Robinson 74MN168, 45P.

16.6 Doroshkevich et al. 74Soviet Astron. 18, 5.

16.7 Illarionov et al. 74Soviet Astron. 18, 6.

16.8 Chan et al. 75ApJ195, 1.

16.9 Hoyle $75 A p J 196,661$.

16.10 Schwartz 74ApJ194, L139.

16.11 Wall et al. $75 M N 171,9$.
16.12 Wesson $75 A S S 36,363$.

16.13 Dehnen et al. 75ASS36, 473.

16.14 Dubrovioh 75Pisma $A Z h 1,10,3$.

16.15 Wickramasinghe et al. 75ASS35, L9.

17. Theory of Radio Sources

(i) Radiation Theory

17.1 Norman et al. 73AA24, Turbulent $121 . \quad$ plasma

17.2 Windsor et al. 74ApJ190, Inverse plasmon 167.

scattering

17.3 Pomraning $74 A p J 191,183$. Stokes parameters induced processes

17.4 Glesson et al. 74MN168, Synchrotron from 379. truncated powerlaw energy spectrum

17.5 Blandford 75MN170, 551. Amplification of radiation by relativistic particles

17.6 Blandford et al. 75ASS36, Induced Compton 303.

Scattering

17.7 Chugunov et al. 75ASS32, Curvature radiaL7. tion

(ii) Effects of Compton scattering

17.8 Okoye $72 M N 160,339 . \quad$ X-ray emission

17.9 Bonometto et al. 73AL13, Circular Polari193.

zation

17.10 Albano et al. 73ASS21, Synchrotron/ 177.

Black-Body.

17.11 Bergeron et al. 72CIAL Observational $17,583$.

consequences

17.12 Okoye $73 M N 165,413$. X-ray generation in QSS

17.13 Harris et al. 74ApJ188, Magnetic field in 209. clusters of galaxies

17.13a Cocke et al. 75ApJ195, Low-frequency 279.

variability

17.13b Guthrie et al.75MN172, 3C273 jet 85.

17.14 Illarionov et al. 74Soviet Formation and Astron. 18, 4.

17.15 Ochelkov et al. 74Soviet Astron. 18, 6. evolution of radiation spectra

17.16 Stewart $75 A A 41,169$. Strong radiative reaction

17.17 Guthrie et al. 75MN172, 3C273 jet 85.

(iii) Source models

17.18 Ozernoy 72 Soviet Astron. 16,5 .

QSO magnetoid 
17.19 Aizu et al. 71Progr. Theor. Phys. Sup. 49, 228.

17.20 Ozernoy 72 Astron. Tsirk. Nuclei of gala$718,1,3$.

17.21 Pacholczyk et al. 73ApJ 179, 21.

17.22 Pecker et al. 73Nat 240, 338.

17.23 Jones $73 A L 14,47$.

17.24 Mashhoon 73ApJ181, L65.

17.25 Cohen 73ApJ181, 619.

17.26 Jura $73 A p J 181,627$.

17.27 Ptak et al. 73Nat 243, 280.

17.28 Dorschner et al. 72 ASS 19, 263. xies magnetoid Restrictions of possible models Abnormal redshifts

Sgr A West

Absorption redshifts

Absorption lines $3 \mathrm{C} 273$ helium abundance Radially streaming proton model

IR spectra

17.29 Clapp 73Phys. Rev. 7, 345. QSO partially collapsed matter

17.30 Bergeron et al. $73 A A 22$, IR X-ray lumi385 .

17.31 Longair et al. 73MN164, Extended radio 243.

17.32 Andrew 73ApJ186, $\mathrm{L} 3$.

17.32a Rees 75MN171, 53.

17.32b Peterson et al. 75 ApJ $195,88$.

17.33 Bridle et al. 73AL15, 21.

17.34 Peterson et al. 73ApJ 186, 421.

17.35 Stepanyan 73Soviet Astron. 17, 6.

17.36 Ohnishi 73ASS25, 217.

17.37 Ozernoy 73Astron. Tsirk. $804,1$.

17.38 Jones et al. 74ApJ188, 353.

17.39 Jones et al. 74ApJ192, 261.

17.40 Buibidge et al. $74 \mathrm{ApJ}$ 193, 43.

17.41 Condon et al. 74ApJ15, 203.

17.42 Sanders 74 Nat 248, 390.

17.42a Rees $75 M N 171,1$. sources

Models of BL Lac Black holes in radio components Synchrotroninverse Compton Double radio source models Prolonged injection model Model of galactic center

Pulsating active nucleus

Constraints models Canonical nonthermal source

Compact nonthermal sources

Energetics of compact sources

Non-uniform compact sources

Super light velocities

Quasar absorption spectra

(iv) Magnetohydrodynamics source confinement

17.43 De Young $72 A p J 177$, 573.

17.44 Ozernoy et al. 73AL13, 209.

17.45 Mackay $73 M N 162,1$.

Relativistically expanding sources Magnetic dipole radiation Limits to velocity
17.46 De Young et al. 73Comm. Form of extended Astrophys. Space.Phys. sources $5,29$.

17.47 Gull et al. 73Nat 244, 80. Bubble model

17.48 Jaffe et al. 73AA26, Tailed radio 423.

17.49 Christiansen 73MN164, 211. sources gas in clusters of galaxies Multiple explosions

17.50 Okoye $73 M N 165,393$.

17.51 Kurilchik 74 Astron. Tsirk. 806, 5.

M87 jet

'Tailed' radio galaxies

17.52 Rumyantsev 74Astrofiz Gaseous ejections 10,1 .

in active galaxies

17.53 Blandford et al. $74 M N$ 169,395 .

17.54 Gull 74Obs94, 1003.

17.55 Shklovskij 75Pisma AZh $1,7,3$.

Twin-exhaust model

Buoyancy model

Galaxy nucleus-

black hole

18. Statistics of Sources, Counts and Related Topics

18.1 Evans $72 M N 160,407$.

Optical spectral indices

18.2 Rozyezka 72Act.Astr.22, Opt.galaxy 93.

counts around QSOs

18.3 Basu 73NatPS241, 159, Opt. selection 243, 144. effect

18.4 Smi et al. 73ApJ181, L61. Opt.Hubble diagrams

18.5 Mills et al. 73AJP26, 417. 0.41 Molonglo

18.6 Plagemann $73 M N 164$, $303 . \quad 3 C$ Parkes

18.7 Maslowski et al. 73AA28, 289.

18.8 Artynkh et al. 73Soviet Astron. 17, 5. $1.4 \mathrm{~GB}$

18.9 Soltan 73Act.Astr.23, 311. 1.4

18.10 Machalski et al. $74 A A 33$, 357.

\section{$1.4 \mathrm{~GB}$}

18.11 Crovisier et al. 74AA34, $285 . \quad 4.8 \mathrm{~B} 2.1$

18.12 Battistihi et al. $74 A A 35$, Opt.radio B2 93.

18.13 Katgert et al. $74 A A 35$, 393.

18.14 Evans et al. 74Obs94, 45. Opt

18.15 Gillespie $75 M N 170,541$. $1.45 \mathrm{C}$

18.16 Wall et al. $75 M N 171,9.2 .7,5.0$

18.17 Hills et al. 73Annals N.Y. Opt.Hubble.diaAcad.Sci.224, 58. gram.Brightest quasars

18.18 Gorshkov et al. 75Pisma AZh1, 9, 19. 8.6 of separation of components
18.19 Larionov 75Astron. Tsirk. 882, 1.

18.20 Balonek et al. 75ApJ 201, 20. 2.7
18.21 Gibson 75AA39, 377. 
(ii) Interpretation and luminosity functions

18.22 Roeder et al. 72NatPS 240, 104.

18.24 Yahil $724 p J 178,45$.

Anisotropy in distribution radio sources

18.25 Burbidge et al. $72 \mathrm{ApJ}$ $178,583$.

Distribution of redshifts

18.26 Gorshkov et al. 72 Astron. New population Tsirk. 720, $2 . \quad$ of radiosources

18.27 Tovmassian $72 A p J 178$, Spiral galaxies L47.

18.28 Soltan 72Act.Astr. 22, Luminosity 261 . function of Gal.

18.29 Fanti et al. 73AA23, 161. QSOs QSSs

18.30 Carswell $73 M N 162,61$. Distribution of distant sources

18.31 Notni et al. 73AN294, 95.

Luminosity

functions

18.32 Basu et al. 72ASS19, 303.

Redshift dist-

ribution

18.33 Vardanian et al. 72

Determination Soobshch.Byurakan.Obs. of completeness 44, 108.

of lists

18.34 Fanti et al. $73 A A 24,69$. Luminosity function.

18.35 Maslowski 73AA26, 343. Anisotropy

18.36 Parijskij 73Soviet Astron. 17, 4 Evolution

18.37 Burbidge et al. 73ApJ183, Redshift magni759. tude

18.38 Karlsson 73NatPS245,68. Selection effects

18.39 Terebizh 73Astrofiz9, Quasar evolution 209.

18.40 Stannard 73Nat 246, 295. Hubble diagram

18.41 Basu 73Obs93, 229. Selection

18.42 Bell et al. $73 A$ pJ 186,1 . Alternative to cosmological QSO

18.43 Van der Kruit 73ApJ15, Angular diameter 27.

18.44 Petrosian et al. $73 \mathrm{ApJ}$ 186, 403.

distribution

Flux density-

spectral index

18.45 von Hoerner $73 A p J 186$, Source counts 741. and evolution

18.46 Burbidge et al. 73ApJ186, L59.

18.47 Romano 73Mem. Soc. Astron. Italiana, Nuova Ser. 44, 47.

18.48 Longair 74IAU Symp.63, Counts of radio 93.

Intergalactic absorption sources.

18.49 Pauliny-Toth et al. 74 IAU Symp.63,111.

Radio source counts at $\mathrm{cm}$ wav lengths

18.50 Fomalont et al. 74AA36, Improved count 273.

18.51 Maslowski $74 A A 36,395$. Spectral index distribution
18.52 Oven $75 A p J 195,593$. Luminosity function of Abell clusters

18.53 Terebizh 75Astrofiz 11, 1. Schmidt luminosity function of quasars

18.54 Gibson 75AA39, 377. Orientation of double radio sources

18.55 Basu 75ApJ16, 53. Effect misidentification

19. Review Papers etc.

(i) Compendia of Data

19.1 Robertson 73AJP26, All-sky cata$403 . \quad$ logue

19.2 Conway et al. 74MN168, Radio polariza137.

tion of quasars

19.3 Clark et al. 74AJP27, 713. Catalogue of small diameter sources close to galactic plane

19.4 Veron et al. 74AASup18, 309.

Catalogue of extragalactic radio source identifications

19.5 Grandi et al. 74PASP86, 873.

Optical variabili-

ty of quasars

19.6 Khachikian et al. $74 \mathrm{ApJ}$ 192, 581.

19.7 Kraus et al. $75 A J 80,1$.

Atlas Seyfert galaxies Radio spectra, redshifts 179 QSO

19.8 Gallouet et al. 75AASup $19,1$.

Optical positions of bright galaxies

(ii) Reviews

19.9 Sazonov 72Uspekhi Fiz. Nauk108, 583.

Circular polarization

19.10 De Young et al. $73 \mathrm{Comm}$. Extended radio Astrophys.Space Phys.5, sources 29.

19.11 Silves to 73Mem.Soc.Astron. Italiana, Nuova Ser.43, High resolution 599.

methods

19.12 Heeschen 73Trans.IAU 15A, 601.

19.13 Troitskij 73Uspekhi Fiz. Nauk. 109, 771.

Report of Commission 40 IAU

19.14 Kellermann et al. 73Proc. Extragalactic IEEE61, 1174.

radio sources

19.15 Kellermann 73Phys. Today 26, 10.

Extragal. radio sources

19.16 Burbidge 73NatPS246, 17.

19.17 Saslaw et al. 73 Comm. Astrophys. Space Phys. $5,133$.
Problem of redshifts

Statistical properties Extragal. radio sources 


\begin{tabular}{|c|c|c|}
\hline 19.18 & Simon 73 Nat 246, 193. & $\begin{array}{l}\text { Submillimetre } \\
\text { astronomy }\end{array}$ \\
\hline 19.19 & $\begin{array}{l}\text { Hagen-Thorn } 74 \text { Astrofiz } \\
10,1 .\end{array}$ & $\begin{array}{l}\text { Optical polariza- } \\
\text { tion of extragal. } \\
\text { objects }\end{array}$ \\
\hline 19.20 & $\begin{array}{l}\text { Longair(ed) } 74 I A U \text { Symp. } \\
63 .\end{array}$ & $\begin{array}{l}\text { Cosmologica the } \\
\text { ories and } \\
\text { observational } \\
\text { data }\end{array}$ \\
\hline 19.21 & $\begin{array}{l}\text { Arp 74IAU Symp.58, } \\
199 .\end{array}$ & $\begin{array}{l}\text { Non-velocity } \\
\text { redshifts }\end{array}$ \\
\hline 19.22 & $\begin{array}{l}\text { Blandtord } 740 b s .94 \text {, } \\
265 \text {. }\end{array}$ & $\begin{array}{l}\text { Double radio } \\
\text { sources }\end{array}$ \\
\hline 19.23 & $\begin{array}{l}\text { Tritton } 74 \text { Sci.Progr. } 242 \text {, } \\
61,275 \text {. }\end{array}$ & $\begin{array}{l}\text { Quasars and the } \\
\text { redshifts }\end{array}$ \\
\hline
\end{tabular}
19.24 Shakeshaft (ed) 74IAU Symp.58.
19.25 Blandford et al. 75 Contemp.Phys.16, 1.
19.26 Kinman 74IAU Symp. 67,573 .
Formation and dynamics of gala- xies Double radio sources Variable quasars and BL Lac type objects
19.27 Van den Bergh 75JRASC Classification $69,57$.
19.28 Thaddeus $72 A R A A 10$, 305.
of normal gala- xies
Short wave- length microwave background
19.29 Van den Bergh 75JRASC Classification of 69, 105. active galaxies

\section{DEVELOPMENTS IN RADIO ASTRONOMY INSTRUMENTS}

\section{(B. J. Robinson)}

\section{A. Radio Telescopes}

\section{Reflectors}

In 1973 the 100-m telescope of the Max Planck Institut für Radioastronomie in Germany became fully operational (1). It has performed well at wavelengths as short as $1.35 \mathrm{~cm}$. On the CSIRO 64-m telescope in Australia an area $37 \mathrm{~m}$ in diameter has been resurfaced to operate at $\lambda 1.35 \mathrm{~cm}$, and a section $17 \mathrm{~m}$ in diameter resurfaced to operate at $\lambda \geqslant 5 \mathrm{~mm}$. The $43-\mathrm{m}$ telescope at Green Bank, U.S.A. has been converted to Cassegrain optics. At the Arecibo Observatory in Puerto Rico the $305-\mathrm{m}$ spherical reflector has been resurfaced (2) with aluminium panels for operation at $\lambda \geqslant 10 \mathrm{~cm}$. For delay-doppler planetary radar at Arecibo a $30-\mathrm{m}$ reflector has been added to resolve position ambiguities by interferometry.

One sector of the RATAN 600 telescope (3) in the Caucasus is in operation at wavelengths between $2 \mathrm{~cm}$ and $6 \mathrm{~cm}$. At $\lambda 2 \mathrm{~cm}$ the beam is $11^{\prime \prime}$ arc $\times 15^{\prime}$ arc. A Kraus-type reflector (4) $25 \mathrm{~m} \times 2 \mathrm{~m}$ at Gorkii, U.S.S.R. has a $20^{\prime \prime}$ arc beam at $\lambda 2 \mathrm{~mm}$.

Radome-enclosed $13.7-\mathrm{m}$ telescopes for short wavelengths have been built at the Itapetinga Radio Observatory in Brazil (5) and by the Helsinki University of Technology (6). A similar 13.7-m telescope is under construction in the U.S.A. at the University of Massachusetts, while a 20.1-m telescope is being built at the Onsala Space Observatory in Sweden. Design principles for $\mathrm{mm}-\lambda$ telescopes have been discussed by von Hoerner (7), while a novel trolley for accurate measurement of reflector profiles has been built (8).

Numerous small reflectors for $\mathrm{mm}$-waves have been brought into operation. These include a 4.6-m telescope in Canada at the University of British Columbia (9), 1.5-m and 6-m telescopes in Japan at Kisaruzu Technical College and Tokyo Astronomical Observatory, and a $1-\mathrm{m}$ telescope in the U.S.A. at NASA Institute for Space Studies. An $8.5-\mathrm{m}$ telescope is under construction for Bell Telephone Laboratories (U.S.A.) and a 4-m telescope is being built for CSIRO in Australia. Optical telescopes at Lick and Palomar (U.S.A.) and at Siding Spring (Australia) have been used for $\mathrm{mm}-\lambda$ observations during daytime or full-moon periods.

\section{Aperture Synthesis Arrays}

Extensive descriptions of the operation and data handling of aperture synthesis arrays have been published. With the Fleurs Synthesis Telescope in Australia (10-19) observations are now being made at $1415 \mathrm{MHz}$ with a $800-\mathrm{m}$ NS arm, which is being extended to $1200 \mathrm{~m}$ while the $800-\mathrm{m} \mathrm{EW}$ arm is being extended to $1600 \mathrm{~m}$. Details of the Westerbork array in The Netherlands 
have also been given (20-27); lower noise receivers have been fitted at $\lambda 21 \mathrm{~cm}$ and work is in progress to double the baseline to $3 \mathrm{~km}$ by adding two $25 \mathrm{-m}$ dishes. A description has also been published of the 5-element synthesis array (28) at Stanford, U.S.A. The 5-km telescope at Cambridge (29) U.K. has been operated at $15 \mathrm{GHz}$ where tropospheric phase errors are serious. At Cambridge an array of 42 elements at $151 \mathrm{MHz}$ allows synthesis over a $1.5 \mathrm{~km}$ baseline; ionospheric phase errors have proved to be troublesome. A report has been published on the status of construction of the Very Large Array (30) in New Mexico, U.S.A.

At the Dominion Radio Astrophysical Observatory in Canada a synthesis array (31) for the $21-\mathrm{cm}$ hydrogen line is now operating. The Cambridge, U.K. 'half-mile' telescope now has 4 elements with digital correlators.

Blum et al. (32) have searched for optimum synthetic linear arrays of 4 antennas used in 2 , 3,4 or 5 configurations.

\section{Interferometers and Arrays}

In Tasmania a $5 \times 10^{5}-\mathrm{m}^{2}$ array for $2-20 \mathrm{MHz}$ has been built as well as a $3 \times 10^{4}-\mathrm{m}^{2}$ array for $40-160 \mathrm{MHz}$. In Florida, U.S.A., Jupiter studies have been made with an array of $640 \lambda / 2$ dipoles. At Clark Lake (U.S.A.) the 720 conical log-spiral elements of the 'Tee Pee Tee' array $(33,34)$ have been connected to form one beam (ultimately 49 ); operating frequency range is $15-130 \mathrm{MHz}$. Also at Clark Lake the COCOA-CROSS $7 \times 10^{4}-\mathrm{m}^{2}$ array (35) has been built to observe interplanetary scintillations at $34 \mathrm{MHz}$. A $3.5-\mathrm{km}$ interferometer system is being linked to the Ootacamund array (36) in India.

For pulsar observations an array $200 \mathrm{~m} \times 400 \mathrm{~m}$ with 16 simultaneous beams at $102.5 \mathrm{MHz}$ has been built at Pushino, U.S.S.R. At the University of Massachusetts, U.S.A. an array of four 36-m spherical reflectors is used to measure pulsars between 150 and $400 \mathrm{MHz}$.

The 2.7- and 8.1-GHz interferometer at Green Bank, U.S.A. has been extended to a baseline of $35 \mathrm{~km}$ with a radio link to a 14-m dish. The electronics for the original 3-element system have been described (37).

A number of mm-wave interferometers have been built or planned. At Hat Creek, USA two 6-m antennas can be located at 50 stations on a T-shaped track $300 \mathrm{~m} \times 200 \mathrm{~m}$; initial operation has been at $\lambda 13.5 \mathrm{~mm}$. At Bordeaux, France, two $2.5 \mathrm{~m}$ antennas on a $60 \mathrm{~m}$ baseline $(38,39)$ have been used for regular observations of the Sun and Venus. The Nagoya, Japan 8-element interferometer (40) at $\lambda 8 \mathrm{~mm}$ is being rebuilt to give an EW resolution of $40^{\prime \prime}$; a two-dimensional position determination (synthesis) takes $2 \mathrm{~h}$. Massachusetts Institute of Technology, U.S.A. are mounting three $5.5-\mathrm{m}$ dishes (good to $\lambda=5 \mathrm{~mm}$ ) on a $300-\mathrm{m}$ baseline with one dish one a $150-\mathrm{m}$ track.

\section{Radioheliographs}

The radioheliograph at Culgoora, Australia, now operates at three (41) frequencies $-43,80$ and $160 \mathrm{MHz}$; a fourth frequency of $327 \mathrm{MHz}$ is being added. The $7.5 \mathrm{~cm}$ radioheliograph at Tokyokawa, Japan, has been extended to a T-array (42) with 16 elements NS and 34 elements $\mathrm{EW}$; the central element is used for phase correction (43).

\section{V. $V L B I$}

Several accounts of $V L B I$ techniques have been published (44-47). The problems of $V L B I$ measurements at low frequencies have been described (48). Phase closure has been used to reduce instrumental phase effects. An improved and cheaper VLBI terminal (Mk IIC) has been developed in the U.S.A. An intercontinental $V L B I$ array (49) has been proposed.

\section{B. Low-Noise Receivers}

Many observatories are now using cryogenically-cooled parametric amplifiers which offer system noise temperatures of $50 \mathrm{~K}$ to $80 \mathrm{~K}$ depending on input configurations. Lower system 
noise is offered by travelling-wave masers (50). System noise temperatures as low as $15 \mathrm{~K}$ have been achieved with T.W. masers used for spacecraft communication (51); these masers can be gain-stabilized by using a noise-adding radiometer (52). A wideband, tunable reflection maser has been built using a section of ruby-filled waveguide (53); the need for a circulator increases the noise somewhat.

At short $\mathrm{cm}$ and millimetre wavelengths cooled Schottky-barrier mixers followed by cooled parametric amplifiers $(54,55)$ offer noise temperatures of $300 \mathrm{~K}$ to $500 \mathrm{~K}$. Similar noise performance has been obtained at wavelengths as short as $1.3 \mathrm{~mm}$ using an InSb bolometer as a mixer (56) but the bandwidth is less than $5 \mathrm{MHz}$. A comprehensive survey of mm-wave techniques has been published by Penzias and Burrus (57).

Significant progress has been made in the development of Josephson junction mixers and amplifiers $(58,59)$. Another new area under development is the cooling of FET's to give noise temperatures near $100 \mathrm{~K}$.

\section{Spectrometers and Spectrographs}

A 1024-channel digital correlator (60) is in operation by CSIRO in Australia; two-bit correlation has shown the expected increase in sensitivity. Multi-level quantization has been used in a digital cross-correlator (61) at the Dominion Radio Astrophysical Observatory, Canada with 125 delays. An asynchronous correlator has been proposed by Ables.

A spectrometer for studying the fine structure of the Sun has been designed (62) in the U.S.S.R. while another has been built with high time resolution (63). A high-speed digital solar spectrograph (64) is operating at Clark Lake, U.S.A. At Culgoora, Australia a broad-band polarimeter has been built for solar bursts, while a novel spectrograph using electro-optical (65) processing has been installed. This electro-optic spectrograph has been tested (66) on the 64-m telescope at Parkes, Australia for recording broad OH profiles, and shows promise as a mm-wave spectrometer to analyse a $100 \mathrm{MHz}$ band into 512 channels.

In Tasmania Ellis has developed a spectrum-analysis technique with high frequency-and time-resolution (67) by recording the signals on a videotape recorder and playing the tape back at reduced speed.

\section{Other Developments}

Techniques for pulsar de-dispersion, searching, data-handling and display have been described by Taylor (68).

Instrumentation of the Explorer-2 radio astronomy satellite (69) for $25 \mathrm{kHz}$ to $13 \mathrm{MHz}$ has been described.

\section{REFERENCES}

1. Hachenberg, O., Grahl, B.H., and Wielebinski, R.: 1973, Proc. IEEE 61, 1288.

2. La Monde, L.M.: 1974, Science 186, 213; see also: 1975, Sky Telesc. 40, 140.

3. Khaikin, S.E., Kaidanovskii, N.L., Parijskii, Y.N., and Esepkina, N.A.: 1972, Izv. Pulkova No. 188, 3.

4. Kislyakov, A.G., Turchin, V.I., Fogel, A.L., and Cherny shev, V.I.: 1973, Radiophys. Quantum Electron. 16, 1093; Kislyakov, A.G., Chernyshev, V.I., Nosov, A.A., and Shandra, Yu.P.: ibid., p. 1086.

5. Kaufmann, P. and D'Amato, R.: 1973, Sky Telesc. 45, 144.

6. Riihimaa, J.J.: 1974, Ann. Acad. Sci. Fennicae A. VI. Phys. No. 413.

7. Von Hoerner, S.: 1975, Astron. Astrophys. 41, 301.

8. Davis, R.T.: 1975, Microwaves 14, 11 .

9. Shuter, W.L.H. and McCutcheon, W.H.: 1974, J. Roy. Astron. Soc. Canada 68, 301.

10. Christiansen, W.N.: 1973, Proc. IEEE 61, 1266; 1973, Proc. IREE (Aust.) 34, 302.

11. Frater, R.H.: 1973, Proc. IREE 34, 309.

12. Watkinson, A., Wellington, K.J., Mondel, R.H., Harwood, M., and Peterson, C.E.: 1973, Proc. IREE 34, 314.

13. O'Sullivan, J.D., Frater, R.H., Imrie, K.S., and Casse, J.L.: 1973, Proc. IREE 34, 319.

14. Frater, R.H., O'Sullivan, J.D., and Daniels, G.R.: 1973, Proc. IREE 34, 326.

15. Mackay, N.A., Kwong, C.K., Jones, I.G., and Symonds, W.A.: 1973, Proc. IREE 34, 329. 
16. Jackson, P.B.: 1973, Proc. IREE 34, 333.

17. Kwong, C.K., Rosolen, K.R., and Jackson, P.B.: 1973, Proc. IREE 34, 337.

18. Brouw, W.N. and O'Sullivan, J.D.: 1973, Proc. IREE 34, 341.

19. O'Sullivan, J.D. and Brouw, W.N.: 1973, Proc. IREE 34, 347.

20. Baars, J.W.M., van der Brugge, J.F., Casse, J.L., Hamaker, J.P., Sondaar, L.H., Visser, J.J., and Wellington, K.J.: 1973, Proc. IEEE 61, 1258.

21. Högbom, J.A. and Brouw, W.N.: 1974, Astron. Astrophys. 33, 289.

22. Baars, J.W.M. and Hooghoudt, B.G.: 1974, Astron. Astrophys. 31, 323.

23. Casse, J.L. and Muller, C.A.: 1974, Astron. Astrophys. 31, 333.

24. Allen, R.J., Hamaker, J.P., and Wlington, K.J.: 1974, Astron. Astrophys. 31, 71.

25. Weiler, K.W.: 1973, Astron. Astrophys. 26, 403.

26. Raimond, E.: 1974, Astron. Astrophys. Suppl. 15, 525.

27. van Someren Grève, H.W.: 1974, Astron. Astrophys. Suppl. 15, 343.

28. Bracewell, R.N., Colvin, R.S., D’Addadario, L.R., Grebenkemper, C.J., Price, K.M., and Thompson, A.R.: 1973, Proc. IEEE 61, 1249.

29. Ryle, M.: 1975, Science 188, 1071; Rev. Mod. Phys. 47, 557.

30. Heeschen, D.S.: 1975, Sky Telesc. 49, 344.

31. Roger, R.S., Costain, C.H., Lacey, J.D., Landecker, T.L., and Bowers, F. K.: 1973, Proc. IEEE 61, 1270.

32. Blum, E.J., Ribes, J.C., and Biraud, F.: 1975, Astron. Astrophys. 41, 409.

33. Erickson, W.C. and Fisher, J.R.: 1974, Radio Sci. 9, 387.

34. Erickson, W.C.: 1973, Proc. IEEE 61, 1276.

35. Cronyn, W.M., Erskine, F.T., and Shawhan, S.D.: 1975, Bull. Am. Astron. Soc. 7, 414.

36. Swarup, G. and Bagri, D.S.: 1975, Proc. IEEE 61, 1285.

37. Coe, J.R.: 1973, Proc. IEEE 61, 1335.

38. Dellanoy, J., Lacroix, J., and Blun, E.J.: 1973, Proc. IEEE 61, 1282.

39. Bocchia, R. and Poumeyrol, F.: 1974, Solar Phys. 38, 193.

40. Kawabata, K., Ogawa, H., Sofue, Y., and Suzuki, I.: 1974, Publ. Astron. Soc. Japan 26, 387.

41. Sheridan, K.V., Labrum, N.R., and Payten, W.J.: 1973, Proc. IEEE 61, 1312.

42. Tanaka, H.: 1974, Proc. Res. Inst. Atmos. Nagoya 21, 33; 1975, 22, 1; 1975, $22,41$.

43. Ishiguro, M.: 1974, Astron. Astrophys. Suppl. 15, 431.

44. Cohen, M.H.: 1973, Proc. IEEE 61, 1192.

45. Counselman, C.C.: 1973, Proc. IEEE 61, 1225.

46. Moran, J.M.: 1973, Proc. IEEE 61, 1236.

47. Clark, B.G.: 1973, Proc. IEEE 61, 1242.

48. Clark, T.A. and Erickson, W.C.: 1973, Proc. IEEE 61, 1230.

49. Swenson, G.W. and Kellermann, K.I.: 1975, Science 188, 1263.

50. Kollberg, E.L.: 1973, Proc. IEEE 61, 1323.

51. Reid, M.S., Clauss, R.C., Bathker, D.A., and Stelzreid, C.T.: 1973, Proc. IEEE 61, 1330.

52. Yerbury, M.J.: 1975, Rev. Sci. Instr. 46, 169.

53. Clauss, R.C.: 1974, JPL Deep Space Network Report 42-25.

54. Weinreb, S. and Kerr, A.R.: 1973, IEEE J. Solid State Circ. 8, 58.

55. Kerr, A.R.: 1975, IEEE Trans. MTT 23, 781.

56. Philips, T.G. and Jefferts, K.B.: 1973, Rev. Sci. Instr. 44, 1009; 1974, IEEE Trans. MTT 22, 1290.

57. Penzias, A.A. and Burrus, C.A.: 1973, Ann. Rev. Astron. Astrophys. $11,51$.

58. Parrish, P.T. and Chiao, R.Y.: 1974, Appl. Phys. Letters 25, 627.

59. Levinsen, M.T. and Ulrich, B.T.: 1974, IEEE Trans. MTT 22, 1117.

60. Ables, J.G., Cooper, B.F.C., Hunt, A.J., Moorey, G.G., and Brooks, J.W.: 1975, Rev. Sci. Instr. 46, 1284.

61. Bowers, F.K., Whyte, D.A., Landecker, T.L., and Klinger, R.J.: 1973, Proc. IEEE 61, 1339.

62. Belov, I.F. et al.: 1973, Izv. Vuzov Radiophys. 16, 725.

63. Kordev, O.S., Markseev, A.E., Fomichev, V.V., and Chertok, I.M.: 1973, Soviet Astron. 50, 1233.

64. Mosier, S.R. and Fainberg, J.: 1975, Solar Phys. 40, 501.

65. Cole, T.W.: 1973, Astrophys. J. Letters 15, 55; 1973, Proc. IEEE 61, 1321.

66. Cole, T.W. and Ables, J.G.: 1974, Astron. Astrophys. 34, 149.

67. Ellis, G.R.A.: 1973, Australian J. Phys. 26, 253.

68. Taylor, J.H.: 1973, Proc. IEEE 61, 1295.

69. Alexander, J.K., Kaiser, M.L., Novaco, J.C., Grena, F.R., and Weber, R.R.: 1975, Astron. Astrophys. 40, 365.

Y. N. PARIJSKIJ

President of the Commission 